\title{
Adaptación Argentina de Dos Escalas de Dificultades en la Regulación Emocional en Adultos Emergentes Universitarios
}

\section{Argentinian Adaptation of Two Scales of Difficulties in Emotion Regulation in College Emerging Adults}

\author{
Yanina Michelini y Juan Carlos Godoy \\ Facultad de Psicología, Universidad Nacional de Córdoba e Instituto de Investigaciones Psicológicas, \\ Consejo Nacional de Investigaciones Científicas y Técnicas, Argentina
}

\begin{abstract}
Las dificultades en la regulación de emociones (i.e., desregulación emocional) negativas y positivas subyacen a conductas riesgosas en adultos emergentes universitarios. La Escala de Dificultades en la Regulación Emocional (DERS) y la Escala de Dificultades en la Regulación de Emociones Positivas (DERS-P) son instrumentos ampliamente usados para medir desregulación emocional; sin embargo, no poseen versiones en español con propiedades psicométricas consistentes. El objetivo de este estudio fue evaluar las propiedades psicométricas de la DERS y DERS$\mathrm{P}$ en adultos emergentes universitarios argentinos. Por medio de un muestreo no probabilístico accidental, se evaluó una muestra de 392 participantes (64,54\% mujeres) de 18 a 28 años $(M=21,40, D E=2,24)$ de 2 universidades públicas de la ciudad de Córdoba, Argentina. Los análisis factoriales confirmatorios que se realizaron a la estructura factorial original de la DERS y DERS-P mostraron ajustes aceptable y excelente, respectivamente. Para ambas escalas, se observaron valores de consistencia interna (alfa de Cronbach y confiabilidad compuesta) satisfactorios. La DERS y la DERS-P presentaron evidencia de validez convergente con estrategias poco adaptativas y divergente con estrategias adaptativas de regulación emocional cognitiva (medidas con el CERQ). Además, presentaron evidencia de validez concurrente con Urgencia Positiva y Urgencia Negativa (medidas con la escala UPPS-P) y con Afecto Positivo y Afecto Negativo (medidos con la PANAS). Extender estos resultados en muestras de estudiantes de distintas carreras universitarias incluyendo la examinación de la confiabilidad test-retest contribuiría a la validación de la DERS y la DERS-P en adultos emergentes universitarios de Argentina.
\end{abstract}

Palabras clave: desregulación emocional, DERS, adaptación argentina, análisis factorial confirmatorio

\begin{abstract}
Difficulties in negative and positive emotion regulation (i.e., emotion dysregulation) underlie risky behaviors in college emerging adults. The Difficulties in Emotion Regulation Scale (DERS) and the Difficulties in Emotion Regulation Scale-Positive (DERS-P) are widely used to measure emotion dysregulation; however, they lack Spanish-language versions with consistent psychometric properties. This study aimed to evaluate the psychometric properties of the DERS and the DERS-P in Argentinian college emerging adults. An accidental non-probability sampling process was conducted, yielding a sample of 392 participants $(64.54 \%$ women) aged 18 to 28 years $(M=21.40 ; S D=2.24)$ who attended 2 public universities in the city of Córdoba, Argentina. Confirmatory factor analyses of the original factor structure of the DERS and the DERS-P revealed excellent and satisfactory fit respectively. For both scales, satisfactory internal consistency values (Cronbach's alpha and composite reliability) were observed. The DERS and the DERS-P exhibited evidence of convergent validity with maladaptive strategies and divergent validity with adaptive strategies of cognitive emotion regulation (assessed with the CERQ). Moreover, they exhibited evidence of concurrent validity with Positive Urgency and Negative Urgency (assessed with the UPPS-P scale) and with Positive Affect and Negative Affect (assessed with the PANAS). Replicating these results in samples of students enrolled in a variety of college programs, incorporating the examination of test-retest reliability, might contribute to the validation of the DERS and the DERS-P in college emerging adults in Argentina.
\end{abstract}

Keywords: emotion dysregulation, DERS, Argentinian validation, confirmatory factor analysis

Yanina Michelini iD https://orcid.org/0000-0003-0768-7645

Juan Carlos Godoy iD https://orcid.org/0000-0002-1622-1647

Los autores agradecen al Dr. Mauricio Federico Zalazar-Jaime, quien colaboró activamente en el análisis de los datos del presente estudio. Este trabajo fue posible gracias a los financiamientos otorgados por el Consejo Nacional de Investigaciones Científicas y Técnicas, en la forma de una beca de doctorado otorgada a la primera autora, y por la Secretaría de Ciencia y Tecnología de la Universidad Nacional de Córdoba, mediante el subsidio de investigación otorgado al proyecto PID 2016-2018, categoría A, titulado "Adolescencia, Cognición y Emoción" y dirigido por el segundo autor. No existe ningún conflicto de intereses que revelar.

La correspondencia relativa a este artículo debe ser dirigida a Yanina Michelini, Facultad de Psicología, Universidad Nacional de Córdoba, Instituto de Investigaciones Psicológicas, Consejo Nacional de Investigaciones Científicas y Técnicas, Bv. de La Reforma esq. Enfermera Gordillo, Estafeta Postal 32, Ciudad Universitaria, Código Postal 5000, Córdoba, Argentina. Email: yaninamichelini@unc.edu.ar 
Cuando se experimentan emociones (i.e., cambios cognitivos, fisiológicos, motivacionales, motores, [LeDoux \& Brown, 2017]), pueden activarse mecanismos por los cuales estas son conducidas hacia fines adaptativos, esto es, reguladas. La regulación emocional se refiere a procesos intrínsecos y extrínsecos para monitorear, evaluar y modificar reacciones emocionales con el fin de lograr objetivos. En este sentido, regular adaptativamente las emociones implica modularlas, es decir, reducir la urgencia asociada a una emoción (Thompson, 1994). La regulación emocional no siempre es adaptativa. La literatura entrega evidencia de que la regulación emocional cumple un papel relevante en cuadros psicopatológicos afectivos y en comportamientos riesgosos (Gross \& Jazaieri, 2014).

Partiendo de aportes conceptuales y empíricos precedentes, Gratz y Roemer (2004) propusieron una definición multidimensional de la regulación emocional. Así, entonces, regular una emoción se caracteriza por las siguientes dimensiones: (a) uso flexible de estrategias adaptativas para modular la intensidad y/o las características temporales de una respuesta emocional; (b) habilidad para resistir conductas impulsivas e involucrarse en comportamientos dirigidos hacia un objetivo; (c) aceptación, claridad y conciencia emocional; y (d) propensión a experimentar angustia emocional cuando se realizan actividades significativas. Desde esta perspectiva, la ausencia relativa de una o más de estas dimensiones indicaría la presencia de dificultades en la regulación emocional, esto es, desregulación emocional.

La adultez emergente es la etapa del desarrollo que transcurre, en general, entre los 18 y los 25 años, aunque puede extenderse hasta los 29 años (Arnett et al., 2014). Los adultos emergentes presentan características que los distinguen de otros grupos etarios. Ya no son adolescentes, pues exhiben madurez física y sexual, se encuentran finalizando la educación formal y alcanzando la mayoría de edad ante la ley. A su vez, desarrollan trayectorias sociales, laborales y educativas más inestables que adultos mayores (Arnett et al., 2014; Skidmore et al., 2016). Con frecuencia, los adultos emergentes se involucran en comportamientos riesgosos, como el consumo problemático de alcohol (i.e., consumo asociado a consecuencias negativas como perder el conocimiento, conducir en estado de ebriedad, tener dificultades para limitar el consumo; Bravo et al., 2019), conductas sexuales sin protección (Organización Panamericana de la Salud, 2017), así como actos delictivos y consumo de sustancias psicoactivas ilegales (Stone et al., 2012; Sussman \& Arnett, 2014). Estas conductas de riesgo, que suelen tener inicio en la adolescencia, se mantienen e incluso aumentan durante la adultez emergente (Mahalik et al., 2013; Shulman \& Cauffman, 2014; Willoughby et al., 2013). Distintos modelos neurobiológicos del desarrollo explican que en la base de esta propensión hacia comportamientos riesgosos se encuentran conexiones cerebrales implicadas en la regulación emocional y la toma de decisiones aún en maduración (Casey et al., 2019; Ernst, 2014). Hasta alcanzar niveles de estabilidad y sofisticación, los procesos de regulación emocional, aun durante la adultez emergente, pueden permanecer rudimentarios y específicos a cada contexto emocional (Thompson, 2011; Zimmermann \& Iwanski, 2014).

Al menos la mitad de los adultos emergentes de numerosos países occidentales, entre ellos, Argentina, inician estudios universitarios durante esta etapa (Organización para la Cooperación y el Desarrollo Económico, 2018). Distintos elementos de la vida universitaria (i.e., nuevos horarios y obligaciones académicas, responsabilidades económicas y nuevos grupos de interacción social, inicio de un empleo al mismo tiempo que se cursa una carrera universitaria [Arnett et al., 2014; Schulenberg \& Maggs, 2002]), sumados a las características propias del desarrollo de la adultez emergente, vuelven particularmente vulnerable a esta subpoblación al involucramiento en conductas de riesgo (Skidmore et al., 2016). Incluso, en varios trabajos se encuentra que adultos emergentes universitarios exhiben mayores comportamientos riesgosos, en comparación con adolescentes y adultos emergentes no universitarios (Bjork \& Pardini, 2015; Skidmore et al., 2016; Stone et al., 2012; Willoughby et al., 2013). Por otro lado, una serie de hallazgos demuestran que las dificultades en la regulación emocional son un factor de riesgo para el desarrollo de trastornos alimentarios (Ambwani et al., 2014; Haynos et al., 2018; Hunt et al., 2017; Miller \& Racine, 2020), consumo problemático de alcohol y otras drogas (Dvorak et al., 2014; Emery et al., 2014; Miller \& Racine, 2020; Simons et al., 2017; Weiss et al., 2018; Zapolski et al., 2009), adicción a internet (Tsai et al., 2020) y conductas sexuales riesgosas (Miller \& Racine, 2020; Zapolski et al., 2009) en adultos emergentes universitarios.

En Argentina, la adultez emergente coincide ampliamente con el cursado de una carrera universitaria. Por ejemplo, el 87\% de los estudiantes de la Universidad Nacional de Córdoba tiene entre 18 y 29 años (Secretaría de Políticas Universitarias, 2017). No obstante, la vida universitaria argentina difiere, en varios aspectos, de las características que presentan las universidades de países desarrollados, desde donde, en general, se origina la mayor parte de la evidencia científica (Chiou, 2001; Lorant et al., 2013). En este sentido, resulta importante que el estudio de los factores de vulnerabilidad relacionados con comportamientos de 
riesgo en adultos emergentes universitarios atienda las características culturales específicas de cada contexto (Arnett, 2015; Bravo et al., 2017).

En el marco de la perspectiva multidimensional de la regulación emocional, Gratz y Roemer (2004) desarrollaron la Escala de Dificultades en la Regulación Emocional (DERS, por sus siglas en inglés), que contiene seis dimensiones o subescalas: (a) Dificultades en el control de impulsos (Impulsos), que se refiere a las dificultades para controlar el propio comportamiento al experimentar emociones negativas; (b) Acceso limitado a estrategias de regulación emocional (Estrategias), que implica la creencia de que, bajo estados emocionales negativos, poco se puede hacer para regular las emociones de manera efectiva; (c) Falta de aceptación emocional (Aceptación), que es la tendencia a tener respuestas emocionales secundarias (e.g., culpa, vergüenza) a las emociones negativas experimentadas o respuestas de no aceptación del malestar provocado por las emociones negativas; (d) Interferencia en conductas dirigidas a metas (Metas), que alude a las dificultades para concentrarse y realizar tareas cuando se experimentan emociones negativas; (e) Falta de conciencia emocional (Conciencia), que refiere a las dificultades para atender y reconocer las emociones que se experimentan y (f) Falta de claridad emocional (Claridad), que alude a las dificultades para conocer y tener en claro las emociones que se experimentan.

Gratz y Roemer (2004) analizaron exploratoriamente una estructura factorial inicial de 41 ítems, luego reducida a 36, en una muestra de estudiantes universitarios. En este estudio, la DERS presentó un ajuste satisfactorio, con indicadores óptimos de consistencia interna $(\alpha=0,93$ para la escala total) y de confiabilidad test-retest en un periodo de cuatro a ocho semanas $(\rho=0,88, p<0,01)$. Además, los resultados mostraron evidencia de validez convergente y divergente mediante correlaciones de la DERS con medidas de expresividad emocional y de regulación del humor negativo. También se obtuvo evidencia de validez predictiva mediante correlaciones con dos medidas de relevancia clínica: la frecuencia de lesiones autoinfringidas y la frecuencia de abuso por parte de la pareja.

La DERS tiene un uso amplio en diferentes contextos culturales, en el ámbito de la investigación y en el campo clínico aplicado. Al respecto, se conocen múltiples estudios de adaptación en distintos países y regiones. Entre las versiones disponibles en idiomas diferentes del español que han confirmado satisfactoriamente la estructura factorial original de la DERS, se conocen una adaptación italiana (Giromini et al., 2012), dos adaptaciones brasileras (Cancian et al., 2019; Miguel et al., 2017), una adaptación francesa (Côté et al., 2013) y una adaptación griega (Mitsopoulou et al., 2013). Asimismo, Ritschel et al. (2015) realizaron análisis factoriales confirmatorios (AFC) de la estructura original de la DERS en estudiantes universitarios estadounidenses. En este estudio, mediante pruebas de invarianza, se examinó si la escala funcionaba de modo similar entre grupos según el sexo, la edad y la etnia. En un estudio de validación en población coreana (Cho \& Hong, 2013), también se realizó AFC, pero la mejor solución la presentó un modelo de cinco factores (i.e., combinando los factores Conciencia y Claridad). Otros estudios (Coutinho et al., 2010; Rugancı \& Gençöz, 2010; Sighinolfi et al., 2010) analizaron la estructura factorial de la DERS mediante análisis factorial exploratorio (AFE), replicando satisfactoriamente la estructura original.

Entre las adaptaciones de la DERS al idioma español, un estudio (Hervás \& Jódar, 2008) que examinó exploratoriamente su estructural factorial, obtuvo una solución coherente e interpretable de cinco factores y 28 ítems. En este caso, los factores fueron renombrados en: Descontrol, Rechazo, Interferencia, Desatención y Confusión. Más tarde, Guzmán-González et al. (2014) realizaron un AFC de la versión de Hervás y Jódar (2008). En este estudio, se obtuvieron resultados satisfactorios para una versión reducida (25 ítems y cinco factores). Por su parte, Muñoz-Martínez et al. (2016) realizaron una adaptación de la DERS original en población colombiana. No obstante, se obtuvo una solución de dos factores que reunían 15 ítems de los 36 originales. Los resultados de este trabajo no permitieron avanzar en subsiguientes análisis de confiabilidad y validez. En Argentina, se conoce una adaptación de la DERS en población universitaria (Medrano \& Trógolo, 2014). Este estudio hace una contribución a la obtención de instrumentos idóneos para el estudio de la desregulación emocional en universitarios argentinos; sin embargo, se observan limitaciones. En primer lugar, contó con una muestra relativamente pequeña $(n=211)$ que presentó un rango de edad amplio (Rango = 18 a 53 años, $M=22,25, D E=3,64$ ) y con escasa variabilidad. Además, se observó un porcentaje mayor al $5 \%$ de casos perdidos para uno de los ítems, cuyo patrón se asoció a las respuestas dadas en otros dos ítems. En segundo lugar, se realizó un AFE que replicó la estructura factorial original (seis factores), sin embargo, ocho ítems debieron ser eliminados por presentar cargas factoriales en más de un factor. Además, para la subescala Estrategias se obtuvo un valor de consistencia interna por debajo de lo aceptable $(\alpha=0,54)$. Por último, los autores del trabajo indicaron que, si bien se obtuvieron resultados promisorios, más estudios psicométricos de la DERS son necesarios para la obtención de resultados con mayor consistencia. En este 
sentido, destacan la importancia de avanzar en estudios que permitan confirmar la estructura factorial original de la DERS, así como en procedimientos que examinen la estabilidad y el poder predictivo de la escala (Medrano \& Trógolo, 2014).

Pese a la amplia utilidad de la DERS, esta medida se focaliza, especialmente, en las dificultades en la regulación de estados emocionales negativos. La conceptualización y medición de la regulación emocional, en cambio, aplica a las experiencias emocionales tanto negativas como positivas (Gratz \& Roemer, 2004; Weiss, Darosh et al., 2019; Weis et al., 2015). En adultos emergentes universitarios, distintos estudios muestran que la desregulación de las emociones positivas se asocia con comportamientos de consumo problemático de alcohol y otras drogas (Weiss et al., 2018; Weiss, Risi et al., 2019; Zapolski et al., 2009). Al respecto, Weiss et al. (2015) desarrollaron la Escala de Dificultades en la Regulación de Emociones Positivas (DERS-P, por sus siglas en inglés), en base a la DERS, en una muestra de estudiantes universitarios. En este estudio, se examinó exploratoriamente la estructura factorial de la DERS-P. Como resultado, se obtuvo una escala de 13 ítems agrupados en tres factores: (a) Aceptación (i.e., tendencia a adoptar una postura de juicio o evaluación hacia las emociones positivas), (b) Objetivos (i.e., capacidad para continuar con las actividades del momento en el contexto de emociones positivas) y (c) Impulsos (i.e., tendencia a realizar acciones precipitadas o imprudentes en el contexto de experiencias emocionales positivas). Además, se observaron indicadores de consistencia interna adecuados y evidencias de validez de constructo satisfactorias. Más tarde, Weiss, Darosh et al. (2019) examinaron la estructura factorial de la DERS-P mediante AFC. Los resultados de este estudio respaldaron la estructura original de tres factores y mostraron evidencias de validez convergente y discriminante con medidas de desregulación emocional negativa, síntomas de malestar psicológico, impulsividad rasgo, síntomas de estrés postraumático y consumo abusivo de drogas. Adicionalmente, la DERS-P resultó útil para diferenciar grupos con y sin niveles clínicamente significativos de estrés postraumático, depresión y consumo abusivo de alcohol y otras drogas. Por otro lado, los resultados de una reciente adaptación italiana de la DERS-P (Velotti et al., 2020) replicaron su estructura factorial original y ofrecieron evidencia de validez relacionada con alexitimia (i.e., incapacidad para experimentar y procesar emociones) y desregulación emocional negativa.

Los problemas para regular emociones negativas y positivas representan un factor de vulnerabilidad subyacente a distintos comportamientos riesgosos en adultos emergentes universitarios (Miller \& Racine, 2020; Weiss, Risi et al., 2019). La DERS es una escala ampliamente utilizada para medir dificultades en la regulación emocional negativa. No obstante, las versiones en español de este instrumento muestran resultados confirmatorios limitados e indicadores de confiabilidad y validez heterogéneos. Desde el mismo enfoque multidimensional, se desarrolló la DERS-P, que mide desregulación emocional positiva. Sin embargo, no se conocen estudios en Argentina o en la región que hayan examinado sus propiedades psicométricas. Contar con instrumentos de autorreporte idóneos y relevantes culturalmente para la medición de la desregulación emocional sería un aporte de utilidad para conocer el modo en que las dificultades en la regulación emocional se asocian con comportamientos de riesgo en adultos emergentes universitarios de Argentina (Miller \& Racine, 2020; Muñiz et al., 2013; Weiss, Darosh et al., 2019). En este contexto, el presente estudio tuvo por objetivo la evaluación de las propiedades psicométricas de las DERS y DERS-P en una muestra de adultos emergentes universitarios argentinos. Específicamente, se realizaron contrastaciones de la validez de constructo y análisis de la consistencia interna de ambas escalas. Además, se examinó la evidencia de validez relacionada con estrategias de regulación emocional cognitiva, urgencia positiva y negativa, afecto positivo y negativo. El presente trabajo pretendió avanzar sobre algunas limitaciones señaladas en los estudios originales de la DERS (Gratz \& Roemer, 2004) y la DERS-P (Weiss, Darosh et al., 2019; Weiss et al., 2015), así como en un estudio en Argentina de validación de la DERS en estudiantes universitarios de Córdoba (Medrano \& Trógolo, 2014). Así, entonces, se conformó una muestra más amplia que en el estudio de Medrano y Trógolo (2014), que incluyó a adultos emergentes universitarios de 18 a 30 años. Además, se examinó la estructura factorial de la DERS mediante un AFC y se avanzó en la adaptación al idioma español de la DERS-P. Otras recomendaciones señaladas en la literatura, como adoptar una técnica de muestreo probabilístico (Hernández Sampieri et al., 2014), así como analizar la estabilidad del instrumento a lo largo del tiempo y obtener evidencia de validez predictiva (Medrano \& Trógolo, 2014; Weiss, Darosh et al., 2019; Weiss et al., 2015), en cambio, se mantuvieron como limitaciones en el presente estudio. $\mathrm{Al}$ respecto, cabe señalar que las dificultades de acceso y disponibilidad de la muestra a lo largo del tiempo, así como los costos asociados a la implementación de estudios que requieren de numerosos pasos (i.e., traducción, entrevistas cognitivas, estudio de jueces, confirmación), estuvieron entre las principales razones por la que algunas recomendaciones fueron priorizadas sobre otras. 


\section{Método}

De manera previa al presente estudio, se realizaron procedimientos de traducción, entrevistas cognitivas y estudio de jueces de las escalas DERS y DERS-P que se encuentran descritos en el siguiente enlace: https://bit.ly/2J1FmiQ. Se siguieron las directrices para la traducción y adaptación de tests de Muñiz et al. (2013).

\section{Participantes}

El diseño muestral fue no probabilístico accidental. La muestra se formó difundiendo, a través redes sociales y listas de correo electrónico, una invitación a participar de un estudio sobre emociones que consistía en completar un cuestionario online. La invitación estaba dirigida a estudiantes de 18 a 30 años, matriculados en dos universidades públicas (Universidad Nacional de Córdoba [UNC] y Universidad Tecnológica Nacional [UTN]) de la ciudad de Córdoba, Argentina. Participó un total de 487 estudiantes (63,45\% mujeres) de 18 a 34 años $(M=21,45, D E=2,44)$. Si bien se elaboró el cuestionario para reducir la probabilidad de datos faltantes (los participantes recibían un aviso ante respuestas faltantes), el 16\% (77 casos) abandonó el cuestionario antes de terminarlo, dejando al menos, un $20 \%$ del cuestionario sin responder. Siguiendo estudios previos (Winfree et al., 2013), estos casos no se consideraron para la muestra final. Además, se eliminaron 15 casos por no cursar estudios en la UNC o UTN y tres casos por ser mayores de 30 años. La muestra final quedó compuesta por 392 estudiantes (64,54\% mujeres) de 18 a 28 años $(M=21,40, D E=2,24)$. La mayoría de los estudiantes pertenecía a diferentes facultades de la UNC. De ellos, 40,05\% eran de Psicología, 8,67\% de Ciencias Médicas, 7,65\% de Artes, 7,40\% de Ciencias Exactas, Físicas y Naturales, 5,36\% de Lenguas, 3,57\% de Arquitectura y Urbanismo, 3,57\% de Ciencias Sociales, 3,06\% de Ciencias Económicas, 2,30\% de Matemática, Astronomía, Física y Computación, 2,30\% de Filosofía y Humanidades, 2,30\% de Ciencias Químicas, 2,04\% de Ciencias de la Comunicación, $1,02 \%$ de Derecho, 0,51\% de Odontología y 0,51\% de Ciencias Agrarias. Por último, un $9,69 \%$ eran estudiantes de la UTN.

\section{Instrumentos}

\section{Escala de Dificultades en la Regulación Emocional (Difficulties in Emotion Regulation Scale; DERS)}

Esta escala, desarrollada por Gratz y Roemer (2004) en Estados Unidos, consta de 36 ítems que describen dificultades en la regulación de emociones negativas. Los ítems de esta escala se agrupan en seis dimensiones: Dificultades en el control de impulsos (Impulsos; seis ítems; e.g., Experimento mis emociones como abrumadoras y fuera de control), Acceso limitado a estrategias de regulación emocional (Estrategias; ocho ítems; e.g., Cuando estoy molesto/a, creo que voy a seguir así por mucho tiempo), Falta de aceptación emocional (Aceptación; seis ítems; e.g., Cuando estoy molesto/a, me siento culpable por sentirme asî), Interferencia en conductas dirigidas a metas (Metas; cinco ítems; e.g., Cuando estoy molesto/a, me cuesta terminar lo que estoy haciendo), Falta de conciencia emocional (Conciencia; seis ítems; e.g., Presto atención a lo que siento) y Falta de claridad emocional (Claridad; cinco ítems; e.g., No tengo idea de cómo me estoy sintiendo). Para responder, los participantes deben indicar con qué frecuencia $(1=$ casi nunca [0-10\%], $2=a$ veces [11-35\%], $3=$ la mitad de las veces [36-65\%], $4=$ la mayoría de las veces [66-90\%], $5=$ casi siempre [91$100 \%$ ]) experimentan las dificultades descritas en cada ítem. El puntaje total y por cada subescala se obtuvo a partir de la sumatoria de los ítems. Un mayor puntaje se interpreta como mayor desregulación de emociones negativas. La versión original de esta escala presenta valores adecuados de consistencia interna (a entre 0,80 y 0,89 para las subescalas y 0,93 para la escala total) y confiabilidad test-retest. Asimismo, la escala mostró evidencia de validez basada en la estructura interna y en la relación con expresividad emocional, regulación del humor negativo, frecuencia de lesiones auto-infringidas y frecuencia de abuso por parte de la pareja.

\section{Escala de Dificultades en la Regulación de Emociones Positivas (Difficulties in Emotion Regulation Scale-Positive; DERS-P)}

La escala DERS-P, desarrollada por Weiss et al. (2015), es una versión basada en la DERS (Gratz \& Roemer, 2004) que examina las dificultades en la regulación emocional derivadas de la experimentación de emociones positivas. La escala cuenta con 13 ítems que se agrupan en tres dimensiones: Falta de aceptación emocional (Aceptación; cuatro ítems; e.g., Cuando estoy contento/a, me asusto y tengo miedo de esas emociones), Interferencia en conductas dirigidas a metas (Metas; cuatro ítems; e.g., Cuando estoy contento /a, 
me cuesta enfocarme en otras cosas) y Dificultades en el control de impulsos (Impulsos; cinco ítems; Cuando estoy contento/a, me preocupa que pueda perder el control). Para responder, los participantes deben indicar con qué frecuencia (desde $1=$ casi nunca [0-10\%] hasta $5=$ casi siempre [91-100\%]) experimentan las dificultades descritas en cada ítem. El puntaje total y por cada subescala se obtuvo a partir de la sumatoria de los ítems. Un mayor puntaje se interpreta como mayor desregulación de emociones positivas. La versión original de esta escala presenta valores adecuados de consistencia interna: a entre 0,83 y 0,87 para las subescalas y 0,90 para la escala total. Asimismo, Weiss, Darosh et al. (2019) obtuvieron evidencia de validez basada en la estructura interna y en la relación con desregulación emocional negativa, síntomas de malestar psicológico, impulsividad rasgo, síntomas de estrés postraumático y consumo abusivo de drogas.

\section{Cuestionario de Regulación Emocional Cognitiva}

Se utilizó la versión en español validada en Argentina (Medrano et al., 2013) de la Cognitive Emotion Regulation Questionnaire (CERQ; Garnesfski et al., 2001). Este cuestionario contiene 36 ítems que examinan el uso de nueve estrategias cognitivas (cuatro poco adaptativas y cinco adaptativas; cuatro ítems por cada estrategia) de regulación emocional frente a una experiencia displacentera o estresante. Las estrategias poco adaptativas son: Rumiación (e.g., Estoy preocupado/a por lo que siento y pienso acerca de lo que me ha pasado), Catastrofización (e.g., Normalmente pienso que lo que me ha pasado a mí es lo peor que le puede pasar a alguien), Autoculparse (e.g., Siento que yo soy el/la culpable de lo que pasó) y Culpar a Otros. Las estrategias adaptativas son: Poner en perspectiva (e.g., Pienso que no ha sido tan malo comparado con otras cosas), Aceptación (e.g., Pienso que tengo que aceptar lo que pasó), Focalización Positiva (e.g., Pienso en cosas positivas que no tienen nada que ver con lo que he vivido), Reinterpretación Positiva (e.g., Pienso que esa situación tiene también partes positivas) y Refocalización en los Planes (e.g., Pienso en cómo cambiar la situación). Para responder, los participantes deben indicar la frecuencia (desde $1=$ casi nunca hasta $5=$ casi siempre) con la que pensó o sintió lo que describe cada ítem. El puntaje por cada subescala se obtuvo a partir de la sumatoria de los ítems. Un mayor puntaje se interpreta como mayor uso de estrategias de regulación emocional cognitiva. Medrano et al. (2013) reportaron valores de consistencia interna no aceptables ( $\alpha$ entre 0,59 y 0,69) para las subescalas Aceptación, Refocalización en Planes, Catastrofización y Autoculparse; mientras que para el resto de las subescalas obtuvieron valores aceptables y safisfactorios ( $\alpha$ entre $0,70 \mathrm{y}$ 0,83). El presente estudio, encontró valores de consistencia interna por fuera de lo aceptable en las subescalas Aceptación, Rumiación y Refocalización en Planes $(\alpha=0,61$ a $\alpha=0,68)$ y valores aceptables o satisfactorios para el resto de las subescalas $(\alpha=0,76$ a $\alpha=0,84)$. Además, la validación argentina del CERQ presentó evidencia de validez basada estructura interna y en la relación con emociones positivas, emociones negativas e interferencia emocional.

\section{Subescalas Urgencia Negativa y Urgencia Positiva de la Escala de Impulsividad (UPPS-P)}

Se utilizó la versión española (Verdejo-García et al., 2010) de las subescalas Negative Urgency y Positive Urgency (UPPS-P Scale; Lynam et al., 2006), que denotan rasgos de la impulsividad. La subescala Urgencia Negativa (12 ítems; e.g., Con frecuencia empeoro las cosas porque actúo sin pensar cuando estoy irritado) evalúa la tendencia a ceder a los impulsos fuertes, especialmente cuando se acompañan de emociones negativas, como la depresión, la ansiedad o la ira. La subescala Urgencia Positiva (14 ítems; e.g., Tiendo a perder el control cuando estoy de muy buen humor) mide la tendencia a ceder a los impulsos en contextos de emoción positiva intensa. Para responder, los participantes deben indicar su grado de acuerdo (desde $1=$ completamente de acuerdo hasta $4=$ completamente en desacuerdo) con lo que describe cada ítem. El puntaje por cada subescala se obtuvo a partir de la sumatoria de los ítems. Un mayor puntaje se interpreta como mayor urgencia negativa o positiva. Estas subescalas presentaron valores de consistencia interna adecuados, tanto en el estudio original ( $\alpha=0,87$ para Urgencia Negativa y a $=0,93$ para Urgencia Positiva; Verdejo-García et al., 2010) como en el presente estudio ( $\alpha=0,88$ para Urgencia Negativa y a $=0,90$ para Urgencia Positiva). Ambas subescalas presentaron evidencia de validez basada en la estructura interna y en la relación con impulsividad, sensibilidad a la recompensa y sensibilidad al castigo.

\section{Escala de Afecto Positivo y Afecto Negativo}

Se utilizó una versión en español evaluada en Argentina (Caicedo Cavagnis et al., 2018) del Positive and Negative Affect Schedule (PANAS; Watson et al., 1988). Esta versión consta de 18 adjetivos (8 ítems para la subescala Afecto Positivo y 10 para Afecto Negativo) que describen estados emocionales positivos (e.g., 
entusiasmado) y negativos (e.g., nervioso). Para responder, los participantes deben indicar en qué medida (desde $1=$ muy poco o nada hasta 5 = extremadamente) experimentan cada uno de los estados emocionales presentados. El puntaje por cada subescala se obtuvo a partir de la sumatoria de los ítems. Un mayor puntaje se interpreta como mayor afecto positivo o negativo. Las subescalas del PANAS presentaron valores de confiabilidad adecuados, tanto en el estudio original (coeficiente de confiabilidad compuesta de 0,87 para Afecto Positivo y de 0,89 para Afecto Negativo; Caicedo Cavagnis et al., 2018) como en el presente estudio (a $=0,84$ para Afecto Positivo y a = 0,88 para Afecto Negativo). Watson et al. (1988) reportaron evidencia de validez basada estructura interna y en la relación con ansiedad, depresión y estrés psicológico.

\section{Procedimiento}

Todos los procedimientos respetaron los lineamientos éticos para la investigación con seres humanos señalados por la American Psychological Association (2017), la declaración de Helsinki de la Asociación Médica Mundial (2008, Estándar 25) y la ley argentina 25.326 de Protección de los datos personales (2000).

Los datos se recolectaron desde el 18 de noviembre hasta el 14 de diciembre de 2016, mediante un cuestionario online desarrollado en el servidor LimeSurvey ${ }^{\circledR}$ (licencia oficial de la UNC). El cuestionario presentaba los instrumentos en el siguiente orden: DERS, DERS-P, CERQ, Subescalas Urgencia Negativa y Urgencia Positiva, y PANAS. Al acceder al cuestionario, los participantes se encontraban con una hoja de información sobre el estudio, en la que se describía el objetivo de la investigación, la naturaleza voluntaria de la participación y la confidencialidad de las respuestas. Asimismo, se indicó que, entre quienes completaran la encuesta, se sortearían dos premios de 500 pesos argentinos. También se brindaban datos de contacto del equipo de investigación responsable del estudio. Por último, se presentaba el consentimiento informado, que cada participante podía otorgar, al optar por responder el cuestionario. Aunque no se solicitó información que permitiera identificar a los participantes (e.g., nombre o apellido), se los invitó a proporcionar una dirección de correo electrónico para ser contactados en caso de resultar ganadores en el sorteo y/o para verificar la potencial duplicación de participación. Esta información se guardó en archivos separados del resto de las respuestas al cuestionario. Completar el cuestionario tomaba aproximadamente 20 minutos.

\section{Análisis de Datos}

En primer lugar, se hicieron cálculos de media, desviación estándar, asimetría y curtosis por cada ítem. Como criterio para evaluar los índices de asimetría y curtosis se consideraron adecuados los valores inferiores a $\pm 2,00$ (George \& Mallery, 2011). Además, se identificaron los casos atípicos univariados mediante el cálculo de puntuaciones $z$ para cada variable (puntuaciones $z> \pm 3,29$ fueron consideradas atípicas) y multivariados mediante la prueba de distancia de Mahalanobis (puntuaciones $p<0,001$ fueron consideradas atípicas).

En segundo lugar, para aportar evidencia de validez basada en la estructura interna de la DERS y la DERS-P, se realizaron AFC al conjunto de ítems de cada escala. Teniendo en cuenta que los instrumentos poseen una escala de respuesta tipo Likert, se utilizó el estimador de mínimos cuadrados ponderados ajustados por la media y varianza, considerado un método de estimación particularmente adecuado para medidas ordinales, variables con anormalidad y casos con atipicidad (Flora \& Curran, 2004; Muthén et al., 1997).

El ajuste de los modelos fue evaluado mediante el estadístico $\chi^{2}$ y $\chi^{2} /$ grados de libertad ( $g l$ ), la raíz del error cuadrático medio de aproximación (Root Mean Square Error of Approximation; RMSEA), el índice de Tucker-Lewis (Tucker-Lewis Index; TLI), el índice comparativo de Bentler-Bonett (Comparative Fit Index; CFI) y la raíz del residuo cuadrático medio ponderado (Weighted Root Mean Square Residual; WRMR). Aunque lo esperado es que el valor de $p$ asociado a $\chi^{2}$ no sea significativo (lo que prueba el modelo nulo frente al propuesto), se debe tener en cuenta la sensibilidad de esta prueba al tamaño muestral, por lo cual tiende a presentar diferencias en muestras moderadas a grandes (Harlow, 2014). En consecuencia, se incluyó el cálculo de $\chi^{2} / g l$, siendo considerados adecuados valores menores a 3 (Carmines \& McIver, 1981). Para el CFI y TLI, valores $\geq 0,90 \mathrm{y} \geq 0,95$ sugieren un ajuste aceptable y excelente, respectivamente. Para el RMSEA, se consideran adecuados valores entre 0,05 y 0,08, mientras que para el WRMR se consideran adecuados los valores $<1$ ( $\mathrm{Yu}, 2002)$. Para los pesos de regresión estandarizados de cada variable observada en la variable latente (Hair et al., 1979/1999), se consideraron óptimos valores $\geq 0,40$.

Adicionalmente, para cada escala, se realizaron correlaciones $r$ de Pearson entre las subescalas. El tamaño de las correlaciones (Cohen, 1988,1992$)$ se interpretó de la siguiente manera: bajo $(0,10)$, medio $(0,30)$ y alto $(0,50)$. 
En tercer lugar, se analizó la consistencia interna de DERS y de DERS-P, tanto para los puntajes totales como para los de cada subescala. Se calcularon coeficientes alfa de Cronbach (a) y se realizaron análisis de confiabilidad compuesta ( $\rho$; Raykov, 1997), un método de mayor robustez para instrumentos con cargas factoriales variables en cada constructo. La confiabilidad compuesta fue analizada mediante un calculador online (Colwell, 2016), disponible en http://www.thestatisticalmind.com/composite-reliability/. Para ambos indicadores de consistencia interna ( a y $\rho$ ), valores $\geq 0,70$ se interpretaron como aceptables y valores $\geq 0,80$ se consideraron satisfactorios (Hogan, 2003/2004; Nunnally \& Bernstein, 1994).

Por último, se realizaron análisis tendientes a aportar evidencia de validez basada en la relación con otras variables, mediante correlaciones de los puntajes totales y de las subescalas de la DERS y la DERS-P con medidas de regulación emocional cognitiva (CERQ), impulsividad rasgo (UPPS-P) y afecto (PANAS). Las correlaciones directas de la DERS y la DERS-P con estrategias poco adaptativas de regulación emocional cognitiva y de la DERS con la DERS-P se interpretaron como evidencia de validez convergente, mientras que correlaciones inversas de la DERS y la DERS-P con estrategias adaptativas de regulación emocional cognitiva se consideraron evidencia de validez divergente. A su vez, correlaciones directas o inversas de la DERS y la DERS-P con Urgencia Positiva, Urgencia Negativa, Afecto Positivo y Afecto Negativo se interpretaron como evidencia de validez concurrente.

Los AFC se realizaron con el programa Mplus Versión 6.12 (Muthén \& Muthén, 2010). Los análisis de datos restantes se realizaron con el programa SPSS Versión 23.

\section{Resultados}

\section{Análisis Descriptivos de la DERS y la DERS-P}

En la DERS, se encontraron seis casos atípicos univariados $(z>3,29)$ y 13 casos atípicos multivariados $(p<0,001)$. Además, todos los ítems presentaron valores adecuados $( \pm 2,00)$ de asimetría y curtosis. En la DERS-P se encontraron 41 casos atípicos univariados $(z>3,29)$ y 34 casos atípicos multivariados $(p<0,001)$. Adicionalmente, ocho ítems presentaron valores por encima de los adecuados $(>2,00)$ en asimetría y 10 ítems, valores por encima de los adecuados $(>2,00)$ en curtosis (ver Tabla 1). Para ambas escalas, los casos atípicos fueron conservados.

\section{Evidencia de Validez de la DERS Basada en la Estructura Interna de la Escala}

El AFC para la estructura original de seis factores de la escala DERS presentó un ajuste aceptable: $\chi^{2}(579)=1723,43, p<0,001, \chi^{2} / g l=2,98$, CFI $=0,92$, TLI $=0,92$, RMSEA $=0,07,90 \%$ IC $[0,07,0,08]$, WRMR $=1,53$. Los factores correlacionaron directamente entre sí, a excepción de la correlación entre Aceptación y Conciencia, que tuvo una significación marginal $(p=0,054)$. Las correlaciones fueron de tamaño medio y alto, salvo para Conciencia que presentó correlaciones bajas con los otros factores (ver Tabla 2). Todos los reactivos presentaron pesos de regresión estandarizados mayores a 0,40, a excepción del ítem 17 ( 0,35 ; Cuando estoy molesto / a, creo que mis emociones son válidas e importantes), perteneciente al factor Conciencia (ver Figura 1).

$\mathrm{Al}$ revisar los índices de modificación, se observó que el ítem 30 (Cuando estoy molesto/a, comienzo a sentirme muy mal conmigo mismo/a), que pertenece al factor Estrategias, presentó una carga cruzada con el factor Aceptación. Al quitar este ítem de Estrategias e incluirlo en Aceptación, el ajuste del modelo mejoró: $\chi^{2}(579)=1519,07, p<0,001, \chi^{2} / g l=2,62, \mathrm{CFI}=0,94, \mathrm{TLI}=0,93, \mathrm{RMSEA}=0,06,90 \%$ IC $[0,06,0,07]$, $\mathrm{WRMR}=1,41$. Además, la carga factorial del ítem 30 se incrementó de 0,87 a 0,94 y no presentó carga cruzada con el factor original. No obstante, considerando que el modelo inicial presentó un ajuste aceptable, y a fin de mantener la estructura original de la escala, se decidió continuar con los análisis de los datos teniendo en cuenta el primer modelo factorial. 
Tabla 1

Media, Desviación Estándar, Asimetría y Curtosis de cada Ítem de la DERS y la DERS-P

\begin{tabular}{|c|c|c|c|}
\hline & Media $(D E)$ & Asimetría & Curtosis \\
\hline \multicolumn{4}{|l|}{ Ítem DERS } \\
\hline 1. Tengo en claro mis emociones a & $2,38(0,94)$ & 0,38 & $-0,52$ \\
\hline 2. Presto atención a lo que siento a & $2,24(1,04)$ & 0,48 & $-0,70$ \\
\hline 3. Experimento mis emociones como abrumadoras y fuera de control & $2,03(1,08)$ & 0,97 & 0,21 \\
\hline 4. No tengo idea de cómo me estoy sintiendo & $1,80(0,96)$ & 1,19 & 0,91 \\
\hline 5. Me cuesta darle sentido a mis emociones & $2,05(1,02)$ & 0,94 & 0,45 \\
\hline 6. Estoy atento/a a mis emociones a & $2,42(1,16)$ & 0,49 & $-0,74$ \\
\hline 7. Sé exactamente cómo me estoy sintiendo a & $2,55(1,10)$ & 0,39 & $-0,70$ \\
\hline 8. Me importa lo que estoy sintiendo a & $1,88(1,11)$ & 1,28 & 0,85 \\
\hline 9. Estoy confundido/a sobre cómo me siento & $2,21(1,06)$ & 0,83 & 0,13 \\
\hline 10. Cuando estoy molesto/a, reconozco mis emociones a & $2,21(1,15)$ & 0,77 & $-0,29$ \\
\hline 11. Cuando estoy molesto/a, me enojo conmigo mismo/a por sentirme así & $2,59(1,32)$ & 0,38 & $-1,08$ \\
\hline 12. Cuando estoy molesto/a, me abochorna (sentir bochorno) sentirme así & $2,28(1,29)$ & 0,65 & $-0,79$ \\
\hline 13. Cuando estoy molesto/a, me cuesta terminar lo que estoy haciendo & $3,05(1,28)$ & $-0,03$ & $-1,11$ \\
\hline 14. Cuando estoy molesto/a, pierdo el control & $1,88(1,16)$ & 1,30 & 0,72 \\
\hline 15. Cuando estoy molesto/a, creo que voy a seguir así por mucho tiempo & $2,08(1,18)$ & 0,98 & 0,03 \\
\hline 16. Cuando estoy molesto/a, creo que voy a terminar sintiéndome muy triste & $2,16(1,31)$ & 0,92 & $-0,40$ \\
\hline 17. Cuando estoy molesto/a, creo que mis emociones son válidas e importantes a & $2,73(1,16)$ & 0,17 & $-0,94$ \\
\hline 18. Cuando estoy molesto/a, me cuesta enfocarme en otras cosas & $3,31(1,18)$ & $-0,11$ & $-1,08$ \\
\hline 19. Cuando estoy molesto/a, me siento fuera de control & $1,81(1,09)$ & 1,39 & 1,24 \\
\hline 20. Cuando estoy molesto/a, aun así puedo terminar las cosas a & $3,23(1,23)$ & $-0,23$ & $-1,00$ \\
\hline 21. Cuando estoy molesto/a, me siento avergonzado/a conmigo mismo/a por sentirme así & $1,88(1,12)$ & 1,23 & 0,65 \\
\hline 22. Cuando estoy molesto/a, sé que puedo encontrar una forma para sentirme mejor a & $2,67(1,22)$ & 0,28 & $-0,93$ \\
\hline 23. Cuando estoy molesto/a, siento que soy débil & $2,23(1,35)$ & 0,81 & $-0,66$ \\
\hline 24. Cuando estoy molesto/a, siento que puedo mantener bajo control mi comportamiento a & $2,73(1,18)$ & 0,16 & $-0,97$ \\
\hline 25. Cuando estoy molesto/a, me siento culpable por sentirme así & $1,96(1,17)$ & 1,19 & 0,48 \\
\hline 26. Cuando estoy molesto/a, me cuesta concentrarme & $3,20(1,22)$ & 0,11 & $-1,06$ \\
\hline 27. Cuando estoy molesto/a, me cuesta controlar mi comportamiento & $2,08(1,08)$ & 0,83 & $-0,05$ \\
\hline 28. Cuando estoy molesto/a, creo que nada puedo hacer para sentirme mejor & $1,91(1,10)$ & 1,29 & 1,03 \\
\hline 29. Cuando estoy molesto/a, me irrito conmigo mismo/a por sentirme así & $2,18(1,23)$ & 0,96 & $-0,05$ \\
\hline 30. Cuando estoy molesto/a, comienzo a sentirme muy mal conmigo mismo/a & $2,14(1,25)$ & 0,94 & $-0,20$ \\
\hline 31. Cuando estoy molesto/a, creo que sumirme en ese estado es lo único que puedo hacer & $1,90(1,17)$ & 1,22 & 0,49 \\
\hline 32. Cuando estoy molesto/a, pierdo el control sobre mi comportamiento & $1,72(1,02)$ & 1,53 & 1,75 \\
\hline 33. Cuando estoy molesto/a, me cuesta pensar en otra cosa & $2,89(1,18)$ & 0,23 & $-0,97$ \\
\hline 34. Cuando estoy molesto/a, me tomo el tiempo necesario para entender lo que estoy sintiendo realmente a & $3,04(1,31)$ & $-0,05$ & $-1,13$ \\
\hline 35. Cuando estoy molesto/a, necesito mucho tiempo para sentirme mejor & $2,27(1,25)$ & 0,73 & $-0,53$ \\
\hline 36. Cuando estoy molesto/a, siento que mis emociones me sobrepasan & $2,56(1,26)$ & 0,41 & $-0,95$ \\
\hline
\end{tabular}


Tabla 1 (Conclusión)

Media, Desviación Estándar, Asimetría y Curtosis de cada Ítem de la DERS y la DERS-P

\begin{tabular}{|c|c|c|c|}
\hline & Media $(D E)$ & Asimetría & Curtosis \\
\hline \multicolumn{4}{|l|}{ Ítem DERS-P } \\
\hline 1. Cuando estoy contento/a, me cuesta enfocarme en otras cosas & $1,82(1,03)$ & 1,28 & 1,00 \\
\hline 2. Cuando estoy contento/a, me enojo conmigo mismo/a por sentirme así & $1,06(0,36)$ & 7,28 & 60,88 \\
\hline 3. Cuando estoy contento/a, me preocupa que pueda perder el control & $1,20(0,55)$ & 3,37 & 13,39 \\
\hline 4. Cuando estoy contento/a, me siento avergonzado/a conmigo mismo/a por sentirme así & $1,10(0,41)$ & 5,44 & 35,67 \\
\hline 5. Cuando estoy contento/a, pierdo el control & $1,23(0,60)$ & 3,42 & 14,30 \\
\hline 6. Cuando estoy contento/a, me asusto y tengo miedo de esas emociones & $1,21(0,62)$ & 3,65 & 14,46 \\
\hline 7. Cuando estoy contento/a, me cuesta concentrarme & $1,82(0,95)$ & 1,16 & 1,01 \\
\hline 8. Cuando estoy contento/a, me cuesta controlar mi comportamiento & $1,40(0,72)$ & 1,95 & 3,71 \\
\hline 9. Cuando estoy contento/a, me cuesta pensar en otra cosa & $1,89(0,01)$ & 1,10 & 0,58 \\
\hline 10. Cuando estoy contento/a, me siento fuera de control & $1,25(0,63)$ & 3,26 & 12,36 \\
\hline 11. Cuando estoy contento/a, me cuesta terminar las cosas & $1,44(0,75)$ & 1,95 & 4,00 \\
\hline 12. Cuando estoy contento/a, me siento culpable por sentirme así & $1,09(0,42)$ & 5,59 & 39,72 \\
\hline 13. Cuando estoy contento/a, pierdo el control sobre mi comportamiento & $1,21(0,58)$ & 3,57 & 14,91 \\
\hline
\end{tabular}

Nota. a Ítems invertidos. 
Tabla 2

Correlaciones entre los Factores de la DERS

\begin{tabular}{lcccccc}
\hline & Impulsos & Estrategias & Aceptación & Metas & Conciencia & Claridad \\
\hline Impulsos & & & & & \\
Estrategias & $0,64^{* * *}$ & & & & \\
Aceptación & $0,42^{* * *}$ & $0,64^{* * *}$ & & & \\
Metas & $0,53^{* * *}$ & $0,63^{* * *}$ & $0,41^{* * *}$ & & \\
Conciencia & $0,19^{* * *}$ & $0,21^{* * *}$ & 0,10 & $0,13^{* * *}$ & \\
Claridad & $0,38^{* * *}$ & $0,45^{* * *}$ & $0,37^{* * *}$ & $0,34^{* * *}$ & $0,64^{* * *}$ & \\
\hline
\end{tabular}

Nota. $n=392 .{ }^{*} p<0,05 ;{ }^{* *} p<0,01 ; * * * p<0,001$.

\section{Figura 1}

Pesos de Regresión Estandarizados de los Ítems de la DERS

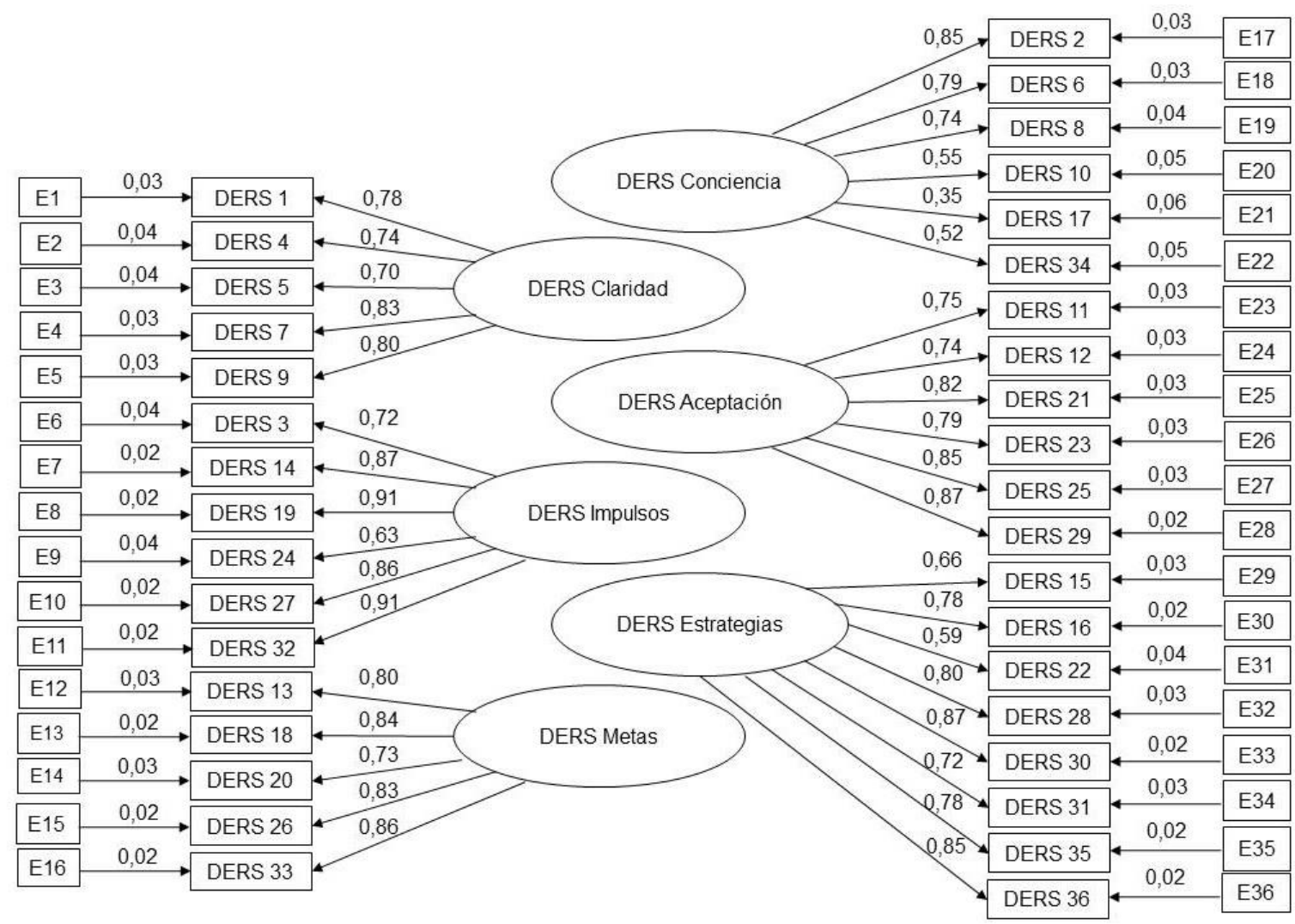

\section{Consistencia Interna de la DERS}

Se obtuvieron índices alfa de Cronbach (a) y confiabilidad compuesta ( $\rho$ ) de 0,87 y 0,93 para Impulsos, 0,89 y 0,92 para Estrategias, 0,88 y 0,92 para Aceptación, 0,88 y 0,91 para Metas, 0,74 y 0,81 para Conciencia, 0,84 y 0,88 para Claridad y 0,93 y 0,98 para la escala total, respectivamente. Si bien se obtuvieron resultados satisfactorios para ambos indicadores de consistencias interna, en todos los casos el valor $\rho$ superó al de $\alpha$, en línea con lo señalado en la literatura (Raykov, 1997). Esta discrepancia resulta esperable si se considera que alfa tiende a subestimar la consistencia interna de una escala, al asumir que las cargas factoriales son las mismas para todos los ítems. En cambio, la confiabilidad compuesta toma en consideración cargas factoriales variables de los reactivos, tal como ocurre en el caso de la DERS. 


\section{Evidencia de Validez de la DERS Basada en la Relación con Otras Variables}

En la Tabla 3 se muestran los resultados de las correlaciones entre los puntajes total y cada subescala de la DERS con los puntajes en las subescalas del CERQ. Se observaron correlaciones directas con las subescalas del CERQ correspondientes a las estrategias poco adaptativas de regulación emocional cognitiva, aportando evidencia de validez convergente. No obstante, Rumiación con Claridad y Conciencia con Autoculparse y Culpar a Otros no mostraron correlación. Además, se observaron correlaciones inversas con las subescalas del CERQ correspondientes a las estrategias adaptativas de regulación emocional, lo que aporta evidencia de validez divergente. No obstante, la subescala Aceptación del CERQ solo correlacionó con la subescala Conciencia de la DERS y las subescalas Poner en Perspectiva y Refocalización en los planes no mostraron correlación con Aceptación de la DERS. Las correlaciones fueron, en general, de tamaño bajo y medio.

Por otro lado, en la Tabla 4 se muestran los resultados de las correlaciones de los puntajes total y cada subescala de la DERS con los puntajes en las subescalas Afecto Positivo y Afecto Negativo del PANAS y con los puntajes en las subescalas Urgencia Positiva y Urgencia Negativa de la Escala UPPS-P. Se observaron correlaciones directas con las subescalas Afecto Negativo, Urgencia Positiva y Urgencia Negativa. Asimismo, se observaron correlaciones inversas con la subescala Afecto Positivo. En general, las correlaciones fueron de tamaño medio. Estos resultados aportan evidencia de validez concurrente.

\section{Evidencia de Validez de la DERS-P Basada en la Estructura Interna de la Escala}

El AFC para la estructura original de tres factores de la escala DERS-P presentó un ajuste excelente: $\chi^{2}(62)=114,75, p<0,001, \chi^{2} / g l=1,85, \mathrm{CFI}=0,99$, TLI $=0,99, \mathrm{RMSEA}=0,05,90 \%$ IC $[0,03,0,06]$, $\mathrm{WRMR}=0,78$. Los factores correlacionaron directamente entre sí. Específicamente, la correlación entre Aceptación y Metas fue de $r(392)=0,31, p<0,01$, la correlación entre Aceptación e Impulsos fue de $r(392)=0,57$, $p<0,01$, y la correlación entre Metas e Impulsos fue de $r(392)=0,53, p<0,01$. Estas correlaciones fueron de tamaño medio y alto. Todos los reactivos presentaron pesos de regresión estandarizados por encima de 0,40 (ver Figura 2).

\section{Consistencia Interna de la DERS-P}

Los resultados mostraron valores satisfactorios de consistencia interna. Específicamente, $\alpha=0,84$ y $\rho=0,93$ para Impulsos, $\alpha=0,78$ y $\rho=0,92$ para Aceptación, $\alpha=0,81$ y $\rho=0,88$ para Metas y $\alpha=0,86$ y $\rho=0,97$ para la escala total. Además, de manera similar a los resultados de confiabilidad de la DERS, se observó que, en todos los casos, el valor de $\rho$ fue mayor que el de $\alpha$.

\section{Evidencia de Validez de la DERS-P Basada en la Relación con Otras Variables}

En relación con la evidencia de validez convergente, se observó que el total y las subescalas de la DERS-P correlacionaron directamente con el total y las subescalas de la DERS, a excepción de la subescala DERS Conciencia, que no correlacionó con DERS-P. Además, la subescala DERS Metas solo correlacionó con la subescala DERS-P del mismo nombre y con DERS-P total. Por otro lado, se obtuvieron correlaciones directas entre la DERS-P y las subescalas del CERQ correspondientes a las estrategias poco adaptativas de regulación emocional. La subescala Culpar a Otros del CERQ correlacionó con el total DERS-P, pero no con las subescalas. Tampoco hubo correlación entre DERS-P Aceptación con CERQ Rumiación y Autoculparse (ver Tabla 5).

En cuanto a la evidencia de validez divergente, se obtuvieron correlaciones inversas y de tamaño pequeño entre las subescalas DERS-P y las estrategias adaptativas de regulación emocional cognitiva. Específicamente, Aceptación, Metas y el total de la DERS-P correlacionaron con Reinterpretación Positiva; Metas y el total de la DERS-P correlacionaron con Focalización Positiva; y Aceptación de la DERS-P correlacionó con Refocalización en los Planes. Las subescalas Poner en perspectiva y Aceptación del CERQ no correlacionaron con las subescalas ni con el total de DERS. Además, DERS-P Impulsos no correlacionó con ninguna de las estrategias adaptativas del CERQ (ver Tabla 5).

Con respecto a la evidencia de validez concurrente, se observaron correlaciones directas de la DERS-P con las subescalas Afecto Negativo de la PANAS y Urgencia Positiva y Urgencia Negativa de la Escala UPPS-P. Además, la DERS-P correlacionó inversamente con la subescala Afecto Positivo de la PANAS con excepción de Impulsos. Las correlaciones, en general, fueron de tamaño medio (ver Tabla 5). 
Tabla 3

Correlaciones del Total y las Subescalas DERS con las Subescalas del CERQ

\begin{tabular}{|c|c|c|c|c|c|c|c|c|c|}
\hline \multirow[b]{2}{*}{ DERS } & \multicolumn{9}{|c|}{ CERQ } \\
\hline & Rumiación & Catastrofización & Autoculparse & Culpar a Otros & $\begin{array}{c}\text { Poner en } \\
\text { Perspectiva }\end{array}$ & Aceptación & $\begin{array}{c}\text { Reinterpretación } \\
\text { Positiva }\end{array}$ & $\begin{array}{l}\text { Focalización } \\
\text { Positiva }\end{array}$ & $\begin{array}{c}\text { Refocalización } \\
\text { en los Planes }\end{array}$ \\
\hline Impulsos & $0,21^{* * *}$ & $0,36^{* * *}$ & $0,26^{* * *}$ & $0,27 * * *$ & $-0,16^{* *}$ & $-0,09$ & $-0,25^{* * *}$ & $-0,22^{* * *}$ & $-0,13^{* *}$ \\
\hline Estrategias & $0,27^{* * *}$ & $0,57 * * *$ & $0,33^{* * *}$ & $0,27 * * *$ & $-0,27 * * *$ & $-0,04$ & $-0,48 * * *$ & $-0,36 * * *$ & $-0,27^{* * *}$ \\
\hline Aceptación & $0,25^{* * *}$ & $0,36^{* * *}$ & $0,37 * * *$ & $0,17 * *$ & $-0,01$ & 0,08 & $-0,18 * * *$ & $-0,10^{*}$ & $-0,07$ \\
\hline Metas & $0,17^{* *}$ & $0,37 * * *$ & $0,21 * * *$ & $0,16^{* *}$ & $-0,22 * * *$ & $-0,05$ & $-0,31 * * *$ & $-0,29 * * *$ & $-0,14^{* *}$ \\
\hline Conciencia & $0,33^{* * *}$ & $0,13^{*}$ & 0,01 & 0,01 & $-0,23^{* * *}$ & $-0,14^{* *}$ & $-0,32 * * *$ & $-0,15^{* *}$ & $-0,33^{* * *}$ \\
\hline Claridad & 0,05 & $0,36 * * *$ & $0,21 * * *$ & $0,18^{* *}$ & $-0,11^{*}$ & $-0,09$ & $-0,27 * * *$ & $-0,20 * * *$ & $-0,19 * * *$ \\
\hline Total & $0,18^{* * *}$ & $0,52^{* * *}$ & $0,34^{* * *}$ & $0,26^{* * *}$ & $-0,24^{* * *}$ & $-0,07$ & $-0,43^{* * *}$ & $-0,32 * * *$ & $-0,26^{* * *}$ \\
\hline
\end{tabular}

Tabla 4

Correlaciones del Total y las Subescalas de la DERS con las Subescalas del PANAS y con las Subescalas de la Escala UPPS-P

\begin{tabular}{lcccc}
\hline \multicolumn{1}{c}{ DERS } & $\begin{array}{c}\text { PANAS } \\
\text { Afecto positivo }\end{array}$ & $\begin{array}{c}\text { PANAS } \\
\text { Afecto negativo }\end{array}$ & $\begin{array}{c}\text { UPPS-P } \\
\text { Urgencia positiva }\end{array}$ & $\begin{array}{c}\text { UPPS-P } \\
\text { Urgencia negativa }\end{array}$ \\
\hline Impulsos & $-0,26^{* * *}$ & $0,56^{* * *}$ & $0,25^{* * *}$ & $0,56^{* * *}$ \\
Estrategias & $-0,43^{* * *}$ & $0,60^{* * *}$ & $0,25^{* * *}$ & $0,50^{* *}$ \\
Aceptación & $-0,28^{* * *}$ & $0,46^{* * *}$ & $0,21^{* * *}$ & $0,35^{* * *}$ \\
Metas & $-0,25^{* * *}$ & $0,45^{* * *}$ & $0,11^{*}$ & $0,43^{* * *}$ \\
Conciencia & $-0,38^{* * *}$ & $0,13^{* *}$ & $0,20^{* * *}$ & $0,19^{* * *}$ \\
Claridad & $-0,42^{* * *}$ & $0,38^{* * *}$ & $0,27^{* * *}$ & $0,38^{* * *}$ \\
Total & $-0,47^{* * *}$ & $0,63^{* * *}$ & $0,30^{* * *}$ & $0,57^{* * *}$ \\
\hline
\end{tabular}

Nota. $n=392,{ }^{*} p<0,05 ; * * p<0,01 ; * * * p<0,001$. 
Figura 2

Pesos de Regresión Estandarizados de los Ítems de la DERS-P
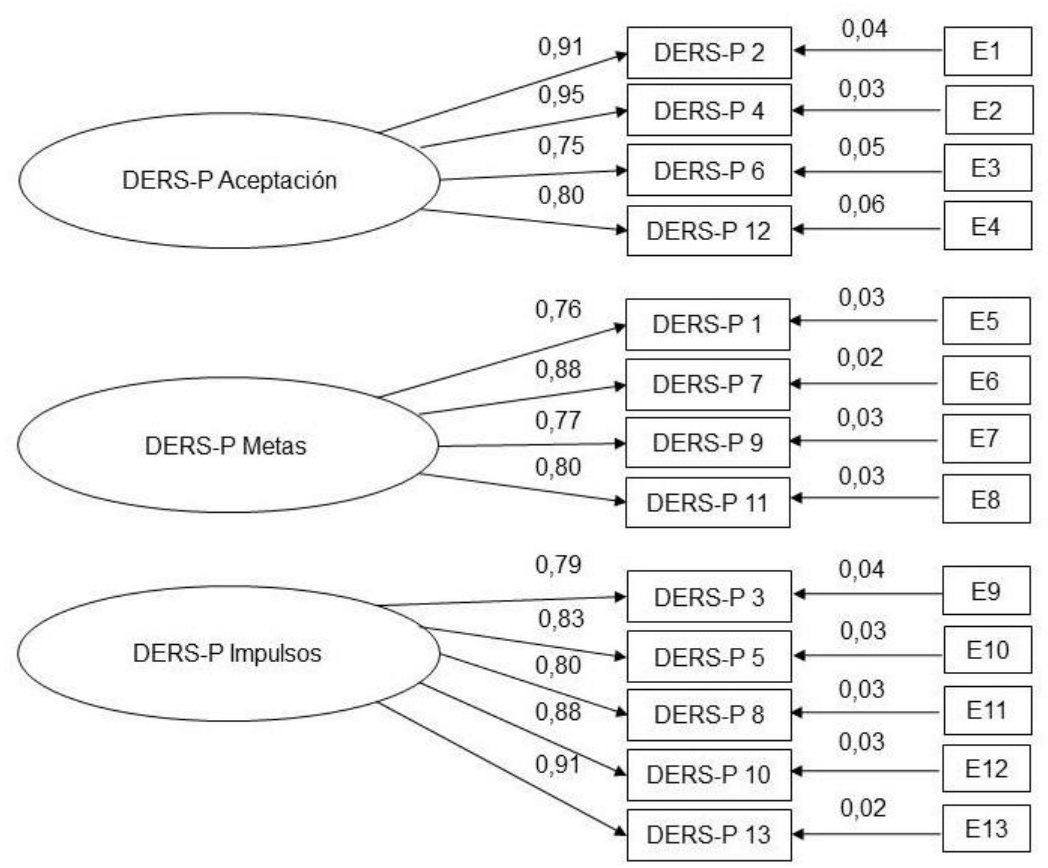

Tabla 5

Correlaciones del Total y las Subescalas de la DERS-P con la DERS, el CERQ, el PANAS y la Escala UPPS-P

\begin{tabular}{|c|c|c|c|c|}
\hline & DERS-P Impulsos & DERS-P Aceptación & DERS-P Metas & DERS-P Total \\
\hline DERS Impulsos & $0,26^{* * *}$ & $0,18^{* *}$ & $0,15^{* *}$ & $0,24 * * *$ \\
\hline DERS Estrategias & $0,23^{* * *}$ & $0,22^{* * *}$ & $0,21^{* * *}$ & $0,27 * * *$ \\
\hline DERS Aceptación & $0,22^{* * *}$ & $0,24^{* * *}$ & $0,15^{* *}$ & $0,24^{* * *}$ \\
\hline DERS Metas & 0,09 & 0,09 & $0,18^{* * *}$ & $0,16^{* * *}$ \\
\hline DERS Conciencia & 0,01 & 0,04 & 0,08 & 0,06 \\
\hline DERS Claridad & $0,20 * * *$ & $0,17^{* *}$ & $0,19^{* * *}$ & $0,23 * * *$ \\
\hline DERS Total & $0,25^{* * *}$ & $0,23^{* * *}$ & $0,23^{* * *}$ & $0,29 * * *$ \\
\hline CERQ Rumiación & $0,14^{* *}$ & 0,01 & $0,11^{*}$ & $0,12^{*}$ \\
\hline CERQ Catastrofización & $0,20 * * *$ & $0,12^{*}$ & $0,19 * * *$ & $0,22^{* * *}$ \\
\hline CERQ Autoculparse & $0,23^{* * *}$ & 0,08 & $0,18^{* * *}$ & $0,22^{* * *}$ \\
\hline CERQ Culpar a Otros & 0,10 & 0,09 & 0,07 & $0,10^{*}$ \\
\hline CERQ Poner en Perspectiva & $-0,03$ & $-0,06$ & $-0,05$ & $-0,05$ \\
\hline CERQ Aceptación & 0,08 & $-0,04$ & 0,04 & 0,05 \\
\hline CERQ Reinterpretación Positiva & $-0,03$ & $-0,12^{*}$ & $-0,12^{*}$ & $-0,11^{*}$ \\
\hline CERQ Focalización Positiva & $-0,07$ & $-0,08$ & $-0,15^{* *}$ & $-0,13 * *$ \\
\hline CERQ Refocalización en los Planes & $-0,01$ & $-0,18 * * *$ & $-0,04$ & $-0,07$ \\
\hline PANAS Afecto Positivo & $-0,06$ & $-0,10^{*}$ & $-0,17^{* *}$ & $-0,14^{* *}$ \\
\hline PANAS Afecto Negativo & $0,33^{* * *}$ & $0,25^{* * *}$ & $0,26^{* * *}$ & $0,35 * * *$ \\
\hline UPPS-P Urgencia Positiva & $0,51^{* * *}$ & $0,33^{* * *}$ & $0,31^{* * *}$ & $0,48^{* * *}$ \\
\hline UPPS-P Urgencia Negativa & $0,23^{* * *}$ & $0,17^{* *}$ & $0,23^{* * *}$ & $0,27 * * *$ \\
\hline
\end{tabular}

Nota. $n=392, * p<0,05 ; * * p<0,01 ; * * * p<0,001$. 


\section{Discusión}

El presente estudio tuvo como objetivo principal la adaptación de la DERS (Gratz \& Roemer, 2004) y de la DERS-P (Weiss et al., 2015) en una muestra de adultos emergentes universitarios argentinos. Los resultados del presente estudio aportan evidencia de validez basada en la estructura interna de ambas escalas y en su relación con dimensiones de regulación emocional cognitiva, impulsividad rasgo y afecto. Además, la DERS y la DERS-P presentaron valores de consistencia interna satisfactorios. A continuación, estos resultados se discuten en mayor detalle.

$\mathrm{Al}$ analizar la estructura interna de la DERS, se observaron resultados aceptables y coherentes con algunos estudios de adaptación previos (Côté et al., 2013; Giromini et al., 2012; Ritschel et al., 2015) en los cuales se realizó un AFC de la estructura original de seis factores. Cabe destacar, además, que todos los reactivos, salvo uno (el ítem 17), presentaron cargas factoriales por encima de 0,40. Por otro lado, al igual que en un estudio previo (Coutinho et al., 2010), el ítem 30, que originalmente pertenece a la subescala Estrategias, cargó favorablemente en el factor Aceptación. Una posible explicación de esta disparidad podría relacionarse con que sentirse muy mal consigo mismo/a no refleje una incapacidad para llevar a cabo una acción (i.e., estrategia) que permita regular las emociones presentes sino, más bien, una reacción emocional secundaria a la emoción negativa vinculada con la falta de aceptación del estrés provocado por la emoción negativa (Gratz \& Roemer, 2004). Sin embargo, aunque sentirse mal puede ser la derivación de una emoción, no implica una emoción en sí misma, como en los casos de tristeza o enojo, que están incluidas como emociones secundarias a la emoción negativa experimentada en reactivos de la subescala Aceptación. En este sentido, y considerando que aunque el ítem cargó en un factor distinto al propuesto, el ajuste del modelo fue aceptable, por lo que se decidió conservar la estructura factorial original de la DERS.

En línea con el estudio original (Gratz \& Roemer, 2004) y otros estudios de adaptación (Cancian et al., 2019; Coutinho et al., 2010; Giromini et al., 2012; Medrano \& Trógolo, 2014; Miguel et al., 2017; Ritschel et al., 2015; Rugancı \& Gençöz, 2010), los resultados de consistencia interna de la DERS fueron, en general, satisfactorios. La subescala Conciencia presentó un alfa menor que el resto de las subescalas $(0,74)$, aunque dentro de límites aceptables (Nunnally \& Bernstein, 1994). Asimismo, mientras que las correlaciones entre las subescalas de la DERS fueron - de acuerdo con lo esperado- de tamaño medio y alto, las correlaciones entre Conciencia y el resto de las subescalas fueron, en general, bajas. Otros estudios sobre las propiedades psicométricas de la DERS han mostrado resultados de confiabilidad y validez dispares para Conciencia, comparada con el resto de las subescalas. En distintas adaptaciones, Conciencia presentó valores aceptables de consistencia interna (Coutinho et al., 2010; Giromini et al., 2012; Rugancı \& Gençöz, 2010), pero también por debajo de límites aceptables (Miguel et al., 2017). A su vez, varios trabajos (Cancian et al., 2019; Giromini et al., 2012; Medrano \& Trógolo, 2014; Mitsopoulou et al., 2013; Ritschel et al., 2015), incluido el estudio que desarrolló la escala (Gratz \& Roemer, 2004), reportaron correlaciones nulas o bajas entre la subescala Conciencia y las otras subescalas de la DERS. Entre las adaptaciones de la DERS al idioma español, un estudio argentino (Medrano \& Trógolo, 2014), que obtuvo un ajuste adecuado de la estructura factorial original, y dos estudios (Guzmán-González et al., 2014; Hervás \& Jódar, 2008) que obtuvieron un ajuste satisfactorio para un modelo de cinco factores, conservaron el factor Conciencia con resultados aceptables de validez y confiabilidad. No obstante, un AFE de la DERS en una muestra colombiana (Muñoz-Martínez et al., 2016) halló una solución de dos factores, uno de ellos, Conciencia y el otro, el conjunto de las subescalas restantes de la versión original de DERS.

Algunas posturas (Cancian et al., 2019; Lee et al., 2016; Muñoz-Martínez et al., 2016; Rugancı \& Gençöz, 2010) señalan que el factor Conciencia no representa estrictamente una dimensión de las dificultades para la regulación emocional, sino que se refiere a dificultades para hacer foco (i.e., centrarse) en las emociones que se experimentan, lo cual representa un paso inicial pero no suficiente para regular una emoción. En consecuencia, desde esta postura se sugiere avanzar en el desarrollo de versiones de la DERS que prescindan del factor Conciencia. Otros autores (Coutinho et al., 2010; Giromini et al., 2012; Hervás \& Jódar, 2008; Mitsopoulou et al., 2013; Ritschel et al., 2015), en cambio, sostienen, de acuerdo con la perspectiva multidimensional de la desregulación emocional, que la conciencia y el entendimiento de las emociones son procesos sustanciales de regulación emocional. Con relación a esto, en su adaptación española de la DERS, Hervás y Jódar (2008) señalaron que las correlaciones más bajas halladas para la subescala Conciencia, respecto a las demás subescalas, eran esperables ya que, comparada con las otras dimensiones, la falta de atención hacia las emociones no tiene un sentido únicamente desadaptativo sino que puede ser un proceso tanto funcional como disfuncional para la regulación emocional. En este sentido, se destaca que la subescala 
Conciencia originalmente presenta propiedades psicométricas menos favorables y mayor independencia respecto de las otras subescalas; no obstante, se mantienen dentro de límites aceptables (Gratz \& Roemer, 2004). Los resultados del presente estudio, de manera similar a otras adaptaciones (Coutinho et al., 2010; Giromini et al., 2012; Mitsopoulou et al., 2013; Ritschel et al., 2015), reflejan estas características de la escala, por lo que se considera pertinente mantener la estructura original de seis factores (que incluye la subescala Conciencia) para la versión de la DERS adaptada en el presente estudio.

$\mathrm{Al}$ examinar la evidencia de validez de la DERS basada en la relación con otras variables, se observaron correlaciones directas con las estrategias poco adaptativas (i.e., rumiación, catastrofización, autoculparse, culpar a otros) y correlaciones inversas con las estrategias adaptativas (i.e., poner en perspectiva, focalización positiva, reinterpretación positiva y refocalización en los planes) de regulación emocional cognitiva. Las correlaciones fueron, en general, de tamaño bajo y medio, indicando que, aunque ambos instrumentos (DERS y CERQ) miden dificultades en la regulación emocional, presentan algunas diferencias. Específicamente, el CERQ examina las estrategias cognitivas que se usan al experimentar un evento negativo (Garnesfski et al., 2001). En cambio, la DERS sirve para indagar dificultades para llevar a cabo procesos tanto cognitivos como conductuales en la regulación de las emociones (Gratz \& Roemer, 2004).

Respecto de la evidencia de validez concurrente de la escala DERS, se observó que mayores dificultades en la regulación emocional se relacionaron con mayor afecto negativo, urgencia positiva y urgencia negativa y con menor afecto positivo. Estos resultados son interesantes si se tiene en cuenta que, durante la adultez emergente, formas inestables y rudimentarias de regular las emociones se vinculan con patrones más exacerbados de experiencia emocional y de impulsividad en contextos emocionales (Casey et al., 2019; Ernst, 2014). Asimismo, distintos estudios indican que las dificultades en la regulación emocional, en conjunto con trastornos del afecto positivo y negativo (Mezquita et al., 2018; Simons et al., 2017; Veilleux et al., 2014) y con la urgencia negativa (Emery et al., 2014), predicen distintos comportamientos de riesgo en adultos emergentes universitarios. Por ejemplo, en un estudio realizado en universitarios de Argentina, España y Estados Unidos (Mezquita et al., 2018), se halló que la regulación del afecto negativo (i.e., consumo de alcohol para aliviar estados afectivos displacenteros) medió el efecto de la inestabilidad emocional sobre el consumo de alcohol y sus consecuencias negativas.

La escala DERS-P, por su parte, presentó valores adecuados de consistencia interna y un ajuste excelente del modelo factorial propuesto. Específicamente, y de manera similar a los estudios previos de validación de esta escala (Weiss, Darosh et al., 2019; Weiss et al., 2015), la consistencia interna presentó valores satisfactorios, con índices de confiabilidad compuesta también mayores que el coeficiente alfa de Cronbach en todos los casos. Las correlaciones entre subescalas tuvieron un tamaño medio a alto. Asimismo, los reactivos presentaron cargas factoriales por encima de 0,40 en el factor correspondiente a la estructura factorial original.

Con respecto a la evidencia de validez basada en la relación con otras variables, se obtuvieron resultados consistentes con lo esperado. En línea con lo observado en los estudios originales (Weiss, Darosh et al., 2019; Weiss et al., 2015), en general, mayores dificultades para regular emociones positivas se relacionaron con mayores dificultades en la regulación emocional negativa. Asimismo, una mayor desregulación emocional en contextos de emociones positivas se relacionó con un mayor uso de estrategias poco adaptativas de regulación emocional cognitiva. También se hallaron algunas correlaciones inversas, entre la DERS-P y las estrategias adaptativas de regulación emocional cognitiva, específicamente, con aquellas que implican realizar reinterpretaciones positivas o focalizarse en pensamientos agradables para aminorar el impacto de un evento negativo (Medrano et al., 2013). La evidencia de validez concurrente, por su parte, mostró correlaciones, en general, de tamaño medio entre la DERS-P y las subescalas de afecto y urgencia. A su vez, similar a lo observado por Weiss Darosh et al. (2019), DERS-P y urgencia positiva correlacionaron fuertemente. Estos resultados son relevantes para el estudio de conductas de riesgo en adultos emergentes universitarios. $\mathrm{Al}$ respecto, estudios muestran que el consumo abusivo de alcohol y de marihuana, de alta prevalencia en universitarios (Pilatti et al., 2017), se vincula estrechamente con la desregulación emocional y con las dificultades para controlar comportamientos impulsivos en el contexto de emociones positivas (Cyders \& Smith, 2007; Weiss et al., 2018; Zapolski et al., 2009). Asimismo, en un reciente estudio realizado en universitarios estadounidenses se encontró que las dificultades para regular emociones positivas moderan la relación entre afecto positivo derivado de estresores sociales (e.g., tener una cita por primera vez, empezar un trabajo) y el consumo de alcohol para afrontar estas situaciones gratificantes pero, a la vez, estresantes (Weiss, Risi et al., 2019). 
Los resultados del presente estudio deben considerarse en el marco de algunas limitaciones. En primer lugar, se aplicó una técnica de muestreo accidental, con la cual se obtuvo una muestra que presentó sobrerrepresentación de mujeres y de estudiantes de psicología. Futuros estudios deberían apelar al uso de técnicas de muestreo más rigurosas (i.e., probabilísticas; Hernández Sampieri et al., 2014), con adecuada distribución por sexo y representación de distintas carreras universitarias. A su vez, sería interesante replicar el estudio en muestras más amplias que permitan la realización de análisis de invarianza en función de características sociodemográficas (Ritschel et al., 2015). En segundo lugar, la evidencia de validez basada en la relación de la DERS y la DERS-P con otras variables solo fue examinada con respecto a otras escalas de autorreporte. En este sentido, es importante considerar que, en general, los instrumentos de evaluación mediante respuesta de autorreporte son vulnerables a los efectos de la deseabilidad social (Eysenck \& Eysenck, 1985/1997). Además, se conoce que algunos procesos de regulación emocional (por ejemplo, aquellos relacionados con la flexibilidad atencional) pueden ocurrir de manera no consciente (Gross, 2015), por lo que el reporte de las dificultades en la regulación de las emociones estaría limitado a acciones conscientes. En consecuencia, podría ser útil que futuras investigaciones examinen evidencia de validez relacionada con pruebas que evalúen el rendimiento comportamental en regulación emocional, por ejemplo, tareas en las cuales los participantes apliquen estrategias de regulación emocional (Silvers et al., 2015). En este sentido, sería importante también examinar evidencia de validez predictiva. Por último, el presente estudio no contó con análisis de confiabilidad test-retest, por lo que se sugiere avanzar en estudios que tengan por objetivo examinar la estabilidad de las escalas DERS y DERS-P.

Más allá de las limitaciones mencionadas, en el presente estudio se obtuvieron datos normativos aceptables y satisfactorios para la medición de las dificultades en la regulación emocional negativa y positiva, respectivamente. Cabe destacar que hasta el momento no se conocían estudios de validación confirmatorios de la escala DERS en población argentina ni adaptaciones de la DERS-P al idioma español. La aplicación de estos instrumentos será de utilidad para conocer el modo en que la desregulación emocional se relaciona con conductas de riesgo en adultos emergentes universitarios argentinos. Más aún, está información permitirá avanzar hacia la identificación de universitarios con mayor propensión a involucrarse en comportamientos que comprometen su salud (Miller \& Racine, 2020; Savolainen et al., 2018), lo que podría derivar en estrategias eficaces de intervención en la regulación de las emociones para la prevención y/o reducción de estos comportamientos (ver, por ejemplo, Sloan et al., 2018). Asimismo, los resultados de este estudio ofrecen un punto de partida para estudios instrumentales y aplicados más específicos como los que se requieren, por caso, para analizar rol de la regulación emocional en cuadros clínicos prevalentes en adultos emergentes, como la ansiedad y la depresión (Arnett et al., 2014; January et al., 2018). Si bien ya hay esfuerzos interesantes en este sentido, el estudio de estas relaciones es aún incipiente en poblaciones de Argentina y de Latinoamérica, por lo que las adaptaciones de la DERS y de la DERS-P resultan un insumo importante.

\section{Referencias}

Ambwani, S., Slane, J. D., Thomas, K. M., Hopwood, C. J. \& Grilo, C. M. (2014). Interpersonal dysfunction and affect-regulation difficulties in disordered eating among men and women. Eating Behaviors, 15(4), 550-554. https://doi.org/10.1016/j.eatbeh.2014.08.005

American Psychological Association. (2017). Ethical principles of psychologists and code of conduct (2003, enmendado el 1 de junio de 2010 y el 1 de enero de 2017). https://www.apa.org/ethics/code/ethics-code-2017.pdf

Arnett, J. J. (2015). Introduction to the special section: Reflections on expanding the cultural scope of adolescent and emerging adult research. Journal of Adolescent Research, 30(6), 655-660. https://doi.org/10.1177/0743558415602556

Arnett, J. J., Žukauskienè, R. \& Sugimura, K. (2014). The new life stage of emerging adulthood at ages 18-29 years: Implications for mental health. The Lancet Psychiatry, 1(7), 569-576. https://doi.org/10.1016/S2215-0366(14)00080-7

Asociación Médica Mundial. (2008). Declaración de Helsinki de la AMM-Principios éticos para las investigaciones médicas en seres humanos (1964, enmendada en octubre de 1975, octubre de 1983, septiembre de 1989, octubre de 1996, octubre de 2000, 2002,2004 y octubre de 2008). https://www.uta.cl/wp-content/uploads/2019/01/Declaracion-de-Helsinki-Asociacion-Medica-Mundial.pdf

Bjork, J. M. \& Pardini, D. A. (2015). Who are those "risk-taking adolescents"? Individual differences in developmental neuroimaging research. Developmental Cognitive Neuroscience, 11, 56-64. https://doi.org/10.1016/j.dcn.2014.07.008

Bravo, A. J., Pearson, M. R., Pilatti, A., Read, J. P., Mezquita, L., Ibáñez, M. I. \& Ortet, G. (2017). Cross-cultural examination of college drinking culture in Spain, Argentina, and USA: Measurement invariance testing of the College Life Alcohol Salience Scale. Drug and Alcohol Dependence, 180, 349-355. https://doi.org/10.1016/j.drugalcdep.2017.08.016

Bravo, A. J., Pilatti, A., Pearson, M. R., Read, J. P., Mezquita, L., Ibáñez, M. I. \& Ortet, G. (2019). Cross-cultural examination of negative alcohol-related consequences: Measurement invariance of the Young Adult Alcohol Consequences Questionnaire in Spain, Argentina, and USA. Psychological Assessment, 31(5), 631-642. https://doi.org/10.1037/pas0000689

Caicedo Cavagnis, E., Michelini, Y., Belaus, A., Mola, D. J., Godoy, J. C. \& Reyna, C. (2018). Further considerations upon PANAS: Contributions from four studies with different Argentinean samples. Suma Psicológica, 25(2), 133-145. https://doi.org/10.14349/sumapsi.2018.v25.n2.5 
Cancian, A. C. M., de Souza, L. A. S., Pesenti e Silva, V. H., Machado, W. d. L. \& Oliveira, M. d. S. (2019). Psychometric properties of the Brazilian version of the Difficulties in Emotion Regulation Scale (DERS). Trends in Psychiatry and Psychotherapy, 41(1), 1826. https://doi.org/10.1590/2237-6089-2017-0128

Carmines, E. G. \& McIver, J. P. (1981). Analyzing models with unobserved variables: Analysis of covariance structures. En G. W. Bohrnstedt \& E. F. Borgatta (Eds.), Social measurement: Current issues (pp. 65-115). SAGE.

Casey, B. J., Heller, A. S., Gee, D. G. \& Cohen, A. O. (2019). Development of the emotional brain. Neuroscience Letters, 693, 29-34. https://doi.org/10.1016/j.neulet.2017.11.055

Chiou, J. -S. (2001). Horizontal and vertical individualism and collectivism among college students in the United States, Taiwan, and Argentina. The Journal of Social Psychology, 141(5), 667-678. https://doi.org/10.1080/00224540109600580

Cho, Y. \& Hong, S. (2013). The new factor structure of the Korean version of the Difficulties in Emotion Regulation Scale (K-DERS) incorporating method factor. Measurement and Evaluation in Counseling and Development, 46(3), 192-201. https://doi.org/10.1177/0748175613484033

Cohen, J. (1988). Statistical power analysis for the behavioral sciences (2 ${ }^{\mathrm{a}}$ ed.). Lawrence Erlbaum.

Cohen, J. (1992). A power primer. Psychological Bulletin, 112(1), 155-159. https://doi.org/10.1037/0033-2909.112.1.155

Colwell, S. R. (2016). The composite reliability calculator. Technical report. The Statistical Mind. https://doi.org/10.13140/RG.2.1.4298.088

Côté, G., Gosselin, P. \& Dagenais, I. (2013). Évaluation multidimensionnelle de la régulation des émotions: Propriétés psychométriques d'une version francophone du Difficulties in Emotion Regulation Scale [Evaluación multidimensional de la regulación de las emociones: propiedades psicométricas de una versión de habla francesa de la Escala de Dificultades en la Regulación Emocional] [Resumen de Science Direct]. Journal de Thérapie Comportementale et Cognitive, 23(2), 63-72. https://doi.org/10.1016/j.jtcc.2013.01.005

Coutinho, J., Ribeiro, E., Ferreirinha, R. \& Dias, P. (2010). The Portuguese version of the Difficulties in Emotion Regulation Scale and its relationship with psychopathological symptoms. Archives of Clinical Psychiatry, 37(4), 152-158. https://doi.org/10.1590/S0101$\underline{60832010000400001}$

Cyders, M. A. \& Smith, G. T. (2007). Mood-based rash action and its components: Positive and negative urgency. Personality and Individual Differences, 43(4), 839-850. https://doi.org/10.1016/j.paid.2007.02.008

Dvorak, R. D., Sargent, E. M., Kilwein, T. M., Stevenson, B. L., Kuvaas, N. J. \& Williams, T. J. (2014). Alcohol use and alcohol-related consequences: Associations with emotion regulation difficulties. The American Journal of Drug and Alcohol Abuse, 40(2), 125-130. https://doi.org/10.3109/00952990.2013.877920

Emery, N. N., Simons, J. S., Clarke, C. J. \& Gaher, R. M. (2014). Emotion differentiation and alcohol-related problems: The mediating role of urgency. Addictive Behaviors, 39(10), 1459-1463. https://doi.org/10.1016/j.addbeh.2014.05.004

Ernst, M. (2014). The triadic model perspective for the study of adolescent motivated behavior. Brain and Cognition, 89, $104-111$. https://doi.org/10.1016/j.bandc.2014.01.006

Eysenck, H. J. \& Eysenck, S. B. G. (1997). Cuestionario Revisado de Personalidad de Eysenck: versión completa (EPQ-R) y abreviada (EPQ-RS). Manual (G. Ortet, M. I. Ibáñez, M.M. Ipola \& F. Silva, adaptadores españoles). TEA. (Obra original publicada en 1985)

Flora, D. B. \& Curran, P. J. (2004). An empirical evaluation of alternative methods of estimation for confirmatory factor analysis with ordinal data. Psychological Methods, 9(4), 466-491. https://doi.org/10.1037/1082-989X.9.4.466

Garnesfski, N., Kraaij, V. \& Spinhoven, P. (2001). Negative life events, cognitive emotion regulation and emotional problems. Personality and Individual Differences, 30(8), 1311-1327. https://doi.org/10.1016/S0191-8869(00)00113-6

George, D. \& Mallery, P. (2011). Descriptive statistics. En S. Hartman (Ed.), SPSS for Windows step by step. A simple guide and reference 18.0 update (pp. 95-104). Pearson.

Giromini, L., Velotti, P., de Campora, G., Bonalume, L. \& Zavattini, G. C. (2012). Cultural adaptation of the Difficulties in Emotion Regulation Scale: Reliability and validity of an Italian version. Journal of Clinical Psychology, 68(9), 989-1007. https://doi.org/10.1002/jclp.21876

Gratz, K. L. \& Roemer, L. (2004). Multidimensional assessment of emotion regulation and dysregulation: Development, factor structure, and initial validation of the Difficulties in Emotion Regulation Scale. Journal of Psychopathology and Behavioral Assessment, 26(1), 41-54. https://doi.org/10.1023/B:JOBA.0000007455.08539.94

Gross, J. J. (2015). Emotion regulation: Current status and future prospects. Psychological Inquiry, 26(1), 1-26. https://doi.org/10.1080/1047840X.2014.940781

Gross, J. J. \& Jazaieri, H. (2014). Emotion, emotion regulation, and psychopathology: An affective science perspective. Clinical Psychological Science, 2(4), 387-401. https://doi.org/10.1177/2167702614536164

Guzmán-González, M., Trabucco, C., Urzúa, A., Garrido, L. \& Leiva, J. (2014). Validez y confiabilidad de la versión adaptada al español de la Escala de Dificultades de Regulación Emocional (DERS-E) en población chilena. Terapia Psicológica, 32(1), 19-29. https://doi.org/10.4067/S0718-48082014000100002

Hair, J. F., Anderson, R. E., Tatham, R. L. \& Black, W. C. (1999). Análisis multivariante (5a ed; E. Prentice \& D. Cano, Trads.). Prentice Hall. (Obra original publicada en 1979)

Harlow, L. L. (2014). The essence of multivariate thinking: Basic themes and methods (2a ed.). Routledge.

Haynos, A. F., Wang, S. B. \& Fruzzetti, A. E. (2018). Restrictive eating is associated with emotion regulation difficulties in a non-clinical sample. Eating Disorders, 26(1), 5-12. https://doi.org/10.1080/10640266.2018.1418264

Hernández Sampieri, R., Fernández Collado, C. \& Baptista Lucio, P. (2014). Metodología de la investigación (6 ed). McGraw Hill Education.

Hervás, G. \& Jódar, R. (2008). Adaptación al castellano de la Escala de Dificultades en la Regulación Emocional. Clínica y Salud, 19(2), 139156. http://scielo.isciii.es/scielo.php?script=sci_arttext\&pid=S1130-52742008000200001\&lng=es\&nrm=iso\&tlng=es

Hogan, T. P. (2004). Pruebas psicológicas: una introducción práctica (J. C. Herrera, Trad.). Manual Moderno. (Obra original publicada en 2003)

Hunt, T. K., Forbush, K. T., Hagan, K. E. \& Chapa, D. A. N. (2017). Do emotion regulation difficulties when upset influence the association between dietary restraint and weight gain among college students? Appetite, 114, 101-109. https://doi.org/10.1016/j.appet.2017.03.029

January, J., Madhombiro, M., Chipamaunga, S., Ray, S., Chingono, A. \& Abas, M. (2018). Prevalence of depression and anxiety among undergraduate university students in low- and middle-income countries: A systematic review protocol. Systematic Reviews, 7, Article 57. https://doi.org/10.1186/s13643-018-0723-8

LeDoux, J. E. \& Brown, R. (2017). A higher-order theory of emotional consciousness. Proceedings of National Academy of Sciences of the United States of America, 114(10), E2016-E2015. https://doi.org/10.1073/pnas.1619316114

Lee, D. J., Witte, T. K., Bardeen, J. R., Davis, M. T. \& Weathers, F. W. (2016). A factor analytic evaluation of the Difficulties in Emotion Regulation Scale. Journal of Clinical Psychology, 72(9), 933-946. https://doi.org/10.1002/jclp.22297 
Lorant, V., Nicaise, P., Soto, V. E. \& d'Hoore, W. (2013). Alcohol drinking among college students: College responsibility for personal troubles. BMC Public Health, 13, Article 615. https://doi.org/10.1186/1471-2458-13-615

Lynam, D. R., Smith, G. T., Whiteside, S. P. \& Cyders, M. A. (2006). The UPPS-P: Assessing five personality pathways to impulsive behavior [Manuscrito no publicado]. Purdue University, Estados Unidos.

Mahalik, J. R., Levine Coley, R., McPherran Lombardi, C., Doyle Lynch, A., Markowitz, A. J. \& Jaffee, S. R. (2013). Changes in health risk behaviors for males and females from early adolescence through early adulthood. Health Psychology, 32(6), 685-694. https://doi.org/10.1037/a0031658

Medrano, L. A., Moretti, L., Ortiz, Á. \& Pereno, G. (2013). Validación del Cuestionario de Regulación Emocional Cognitiva en universitarios de Córdoba, Argentina. Psykhe, 22(1), 83-96. https://doi.org/10.7764/psykhe.22.1.473

Medrano, L. A. \& Trógolo, M. (2014). Validación de la Escala de Dificultades en la Regulación Emocional en la población universitaria de Córdoba, Argentina. Universitas Psychologica, 13(4), 1345-1356. https://doi.org/10.11144/Javeriana.UPSY13-4.vedr

Mezquita, L., Bravo, A. J., Ortet, G., Pilatti, A., Pearson, M. R. \& Ibáñez, M. I. (2018). Cross-cultural examination of different personality pathways to alcohol use and misuse in emerging adulthood. Drug and Alcohol Dependence, 192, 193-200. https://doi.org/10.1016/j.drugalcdep.2018.08.004

Miguel, F. K., Giromini, L., Colombarolli, M. S., Zuanazzi, A. C. \& Zennaro, A. (2017). A Brazilian investigation of the 36- and 16-item Difficulties in Emotion Regulation Scales. Journal of Clinical Psychology, 73(9), 1146-1159. https://doi.org/10.1002/jclp.22404

Miller, A. E. \& Racine, S. E. (2020). Emotion regulation difficulties as common and unique predictors of impulsive behaviors in university students. Journal of American College Health. Anticipo en línea de la publicación. https://doi.org/10.1080/07448481.2020.1799804

Mitsopoulou, E., Kafetsios, K., Karademas, E., Papastefanakis, E. \& Simos, P. G. (2013). The Greek version of the Difficulties in Emotion Regulation Scale: Testing the factor structure, reliability and validity in an adult community sample. Journal of Psychopathology and Behavioral Assessment, 35(1), 123-131. https://doi.org/10.1007/s10862-012-9321-6

Muñiz, J., Elosua, P. \& Hambleton, R. K. (2013). Directrices para la traducción y adaptación de los tests: segunda edición. Psicothema, 25(2), 151-157. https://doi.org/10.7334/psicothema2013.24

Muñoz-Martínez, A. M., Vargas, R. M. \& Hoyos-González, J. S. (2016). Escala de Dificultades en Regulación Emocional (DERS): análisis factorial en una muestra colombiana. Acta Colombiana de Psicología, 19(1), 225-236. https://doi.org/10.14718/ACP.2016.19.1.10

Muthén, B., du Toit, S. H. \& Spisic, D. (1997). Robust inference using weighted least squares and quadratic estimating equations in latent variable modeling with categorical and continuous outcomes [Manuscrito no publicado]. https://www.statmodel.com/download/Article_075.pdf

Muthén, L. K. \& Muthén, B. O. (2010). Mplus: Statistical analysis with latent variables. User's Guide (6 ${ }^{a}$ ed.). https://www.yumpu.com/en/document/read/40845405/mplus-users-guide-v6-muthen-muthen

Nunnally, J. C. \& Bernstein, I. H. (1994). Psychometric theory (3a ed.). McGraw Hill.

Organización Panamericana de la Salud. (2017). Virus de la inmunodeficiencia humana (VIH) e infecciones de transmisión sexual (ITS) en las Américas. https://www.paho.org/salud-en-las-americas-2017/?post_t_es=virus-de-la-inmunodeficiencia-humana-vih-einfecciones-de-transmision-sexual-its-en-las-americas\&lang=fr

Organización para la Cooperación y el Desarrollo Económico. (2018). Education at a glance 2018: OECD indicators. https://doi.org/10.1787/eag-2018-en

Pilatti, A., Read, J. P. \& Pautassi, R. M. (2017). ELSA 2016 cohort: Alcohol, tobacco, and marijuana use and their association with age of drug use onset, risk perception, and social norms in Argentinean college freshmen. Frontiers in Psychology, 8, Artículo 1452. https://doi.org/10.3389/fpsyg.2017.01452

Protección de los datos personales, Ley 25.326, Senado y Cámara de Diputados de la Nación Argentina (2000). https://www.oas.org/juridico/pdfs/arg_ley25326.pdf

Raykov, T. (1997). Estimation of composite reliability for congeneric measures. Applied Psychological Measurement, 21(2), 173-184. https://doi.org/10.1177/01466216970212006

Ritschel, L. A., Tone, E. B., Schoemann, A. M. \& Lim, N. E. (2015). Psychometric properties of the Difficulties in Emotion Regulation Scale across demographic groups. Psychological Assessment, 27(3), 944-954. https://doi.org/10.1037/pas0000099

Rugancı, R. N. \& Gençöz, T. (2010). Psychometric properties of a Turkish version of the Difficulties in Emotion Regulation Scale. Journal of Clinical Psychology, 66(4), 442-455. https://doi.org/10.1002/jclp.20665

Savolainen, I., Kaakinen, M., Sirola, A. \& Oksanen, A. (2018). Addictive behaviors and psychological distress among adolescents and emerging adults: A mediating role of peer group identification. Addictive Behaviors Reports, 7, 75-81. https://doi.org/10.1016/j.abrep.2018.03.002

Schulenberg, J. E. \& Maggs, J. L. (2002). A developmental perspective on alcohol use and heavy drinking during adolescence and the transition to young adulthood. Journal of Studies on Alcohol, Supplement 14, 54-70. https://doi.org/10.15288/jsas.2002.s14.54

Secretaría de Políticas Universitarias. (2017). Sistema de consulta de estadísticas universitarias. Presidencia de la Nación Argentina. http://estadisticasuniversitarias.me.gov.ar/\#/seccion/1

Shulman, E. P. \& Cauffman, E. (2014). Deciding in the dark: Age differences in intuitive risk judgment. Developmental Psychology, 50(1), 167-177. https://doi.org/10.1037/a0032778

Sighinolfi, C., Pala, A. N., Chiri, L. R., Marchetti, I. \& Sica, C. (2010). Difficulties in Emotion Regulation Scale (DERS): Traduzione e adattamento italiano [Escala de Dificultades en la Regulación Emocional (DERS): traducción y adaptación al italiano] [Resumen de APA PsicNet]. Psicoterapia Cognitiva e Comportamentale, 16(2), 141-170. https://psycnet.apa.org/record/2010-15362-001

Silvers, J. A., Shu, J., Hubbard, A. D., Weber, J. \& Ochsner, K. N. (2015). Concurrent and lasting effects of emotion regulation on amygdala response in adolescence and young adulthood. Developmental Science, 18(5), 771-784. https://doi.org/10.1111/desc.12260

Simons, R. M., Hahn, A. M., Simons, J. S. \& Murase, H. (2017). Emotion dysregulation and peer drinking norms uniquely predict alcoholrelated problems via motives. Drug and Alcohol Dependence, 177, 54-58. https://doi.org/10.1016/j.drugalcdep.2017.03.019

Skidmore, C. R., Kaufman, E. A. \& Crowell, S. E. (2016). Substance use among college students. Child and Adolescent Psychiatric Clinics of North America, 25(4), 735-753. https://doi.org/10.1016/j.chc.2016.06.004

Sloan, E., Hall, K., Simpson, A., Youssef, G. J., Moulding, R., Mildred, H. \& Staiger, P. K. (2018). An emotion regulation treatment for young people with complex substance use and mental health issues: A case-series analysis. Cognitive and Behavioral Practice, 25(3), 427-441. https://doi.org/10.1016/j.cbpra.2017.12.006

Stone, A. L., Becker, L. G., Huber, A. M. \& Catalano, R. F. (2012). Review of risk and protective factors of substance use and problem use in emerging adulthood. Addictive Behaviors, 37(7), 747-775. https://doi.org/10.1016/j.addbeh.2012.02.014

Sussman, S. \& Arnett, J. J. (2014). Emerging adulthood: Developmental period facilitative of the addictions. Evaluation \& The Health Professions, 37(2), 147-155. https://doi.org/10.1177/0163278714521812 
Thompson, R. A. (1994). Emotion regulation: A theme in search of definition. Monographs of the Society for Research in Child Development, 59(2-3), 25-52. https://doi.org/10.1111/j.1540-5834.1994.tb01276.x

Thompson, R. A. (2011). Emotion and emotion regulation: Two sides of the developing coin. Emotion Review, 3(1), 53-61. https://doi.org/10.1177/1754073910380969

Tsai, J. -K., Lu, W. -H., Hsiao, R. C., Hu, H. -F. \& Yen, C. -F. (2020). Relationship between difficulty in emotion regulation and internet addiction in college students: A one-year prospective study. International Journal of Environmental Research and Public Health, 17(13), Artículo 4766. https://doi.org/10.3390/ijerph17134766

Veilleux, J. C., Skinner, K. D., Reese, E. D. \& Shaver, J. A. (2014). Negative affect intensity influences drinking to cope through facets of emotion dysregulation. Personality and Individual Differences, 59, 96-101. https://doi.org/10.1016/j.paid.2013.11.012

Velotti, P., Rogier, G., Civilla, C., Garofalo, C., Serafini, G. \& Amore, M. (2020). Dysregulation of positive emotions across community, clinical and forensic samples using the Italian version of the Difficulties in Emotion Regulation Scale-Positive (DERS-Positive). The Journal of Forensic Psychiatry \& Psychology, 31(4), 555-570. https://doi.org/10.1080/14789949.2020.1776374

Verdejo-García, A., Lozano, Ó., Moya, M., Alcázar, M. Á. \& Pérez-García, M. (2010). Psychometric properties of a Spanish version of the UPPS-P Impulsive Behavior Scale: Reliability, validity and association with trait and cognitive impulsivity. Journal of Personality Assessment, 92(1), 70-77. https://doi.org/10.1080/00223890903382369

Watson, D., Clark, L. A. \& Tellegen, A. (1988). Development and validation of brief measures of positive and negative affect: The PANAS scales. Journal of Personality and Social Psychology, 54(6), 1063-1070. https://doi.org/10.1037//0022-3514.54.6.1063

Weiss, N. H., Darosh, A. G., Contractor, A. A., Schick, M. M. \& Dixon-Gordon, K. L. (2019). Confirmatory validation of the factor structure and psychometric properties of the Difficulties in Emotion Regulation Scale-Positive. Journal of Clinical Psychology, 75(7), 12671287. https://doi.org/10.1002/jclp.22768

Weiss, N. H., Forkus, S. R., Contractor, A. A. \& Schick, M. R. (2018). Difficulties regulating positive emotions and alcohol and drug misuse: A path analysis. Addictive Behaviors, 84, 45-52. https://doi.org/10.1016/j.addbeh.2018.03.027

Weiss, N. H., Gratz, K. L. \& Lavender, J. M. (2015). Factor structure and initial validation of a multidimensional measure of difficulties in the regulation of positive emotions: The DERS-Positive. Behavior Modification, 39(3), 431-453. https://doi.org/10.1177/0145445514566504

Weiss, N. H., Risi, M. M., Bold, K. W., Sullivan, T. P. \& Dixon-Gordon, K. L. (2019). Daily relationship between positive affect and drinking to cope: The moderating role of difficulties regulating positive emotions. The American Journal of Drug and Alcohol Abuse, 45(2), 189-198. https://doi.org/10.1080/00952990.2018.1508470

Winfree, W. R., Meyers, A. W. \& Whelan, J. P. (2013). Validation of a Spanish translation of the Gamblers' Beliefs Questionnaire. Psychology of Addictive Behaviors, 27(1), 274-278. https://doi.org/10.1037/a0030824

Willoughby, T., Good, M., Adachi, P. J. C., Hamza, C. \& Tavernier, R. (2013). Examining the link between adolescent brain development and risk taking from a social-developmental perspective. Brain and Cognition, 83(3), 315-323. https://doi.org/10.1016/j.bandc.2013.09.008

Yu, C. -Y. (2002). Evaluating cutoff criteria of model fit indices for latent variable models with binary and continuous outcomes (Tesis de Doctorado no publicada). Universidad de California. http://www.statmodel.com/download/Yudissertation.pdf

Zapolski, T. C. B., Cyders, M. A. \& Smith, G. T. (2009). Positive urgency predicts illegal drug use and risky sexual behavior. Psychology of Addictive Behaviors, 23(2), 348-354. https://doi.org/10.1037/a0014684

Zimmermann, P. \& Iwanski, A. (2014). Emotion regulation from early adolescence to emerging adulthood and middle adulthood: Age differences, gender differences, and emotion-specific developmental variations. International Journal of Behavioral Development, 38(2), 182-194. https://doi.org/10.1177/0165025413515405

Fecha de recepción: Marzo de 2020.

Fecha de aceptación: Febrero de 2021. 